\title{
The Multiple-partners Assignment Game with Heterogeneous Sales and Multi-unit Demands: Competitive Equilibria*
}

\author{
Daniel Jaume ${ }^{\dagger}$, Jordi Massó ${ }^{\ddagger}$, And Alejandro Neme ${ }^{\dagger}$
}

First version: December 2007

This version: December 2009

\begin{abstract}
A multiple-partners assignment game with heterogeneous sales and multiunit demands consists of a set of sellers that own a given number of indivisible units of (potentially many different) goods and a set of buyers who value those units and want to buy at most an exogenously fixed number of units. We define a competitive equilibrium for this generalized assignment game and prove its existence by using only linear programming. In particular, we show how to compute equilibrium price
\end{abstract}

${ }^{*}$ We thank Ester Camiña, John Hatfield, and Alejandro Manelli for their very helpful comments. The work of D. Jaume and A. Neme is partially supported by the Universidad Nacional de San Luis through grant 319502 and by the Consejo Nacional de Investigaciones Científicas y Técnicas (CONICET) through grant PICT-02114. Support for the research of J. Massó was received through the prize "ICREA Acadèmia" for excellence in research, funded by the Generalitat de Catalunya. He also acknowledges the support of MOVE, where he is an affiliated researcher, and of the Barcelona Graduate School of Economics (through its Research Recognition Programme), where he is an affiliated professor. His work is also supported by the Spanish Ministry of Science and Innovation through grants ECO2008-04756 (Grupo Consolidado-C) and CONSOLIDER-INGENIO 2010 (CDS2006-00016), and by the Generalitat de Catalunya through grant SGR2009-419.

†Instituto de Matemática Aplicada de San Luis. Universidad Nacional de San Luis and CONICET. Ejército de los Andes 950. 5700, San Luis, Argentina. E-mails: djaume@unsl.edu.ar and aneme@unsl.edu.ar

${ }_{\ddagger}^{\ddagger}$ Departament d'Economia i d'Història Econòmica and CODE. Universitat Autònoma de Barcelona. 08193, Bellaterra (Barcelona), Spain. E-mail: jordi.masso@uab.es 
vectors from the solutions of the dual linear program associated to the primal linear program defined to find optimal assignments. Using only linear programming tools, we also show (i) that the set of competitive equilibria (pairs of price vectors and assignments) has a Cartesian product structure: each equilibrium price vector is part of a competitive equilibrium with all optimal assignments, and vice versa; (ii) that the set of (restricted) equilibrium price vectors has a natural lattice structure; and (iii) how this structure is translated into the set of agents' utilities that are attainable at equilibrium.

Journal of Economic Literature Classification Numbers: C78; D78.

Keywords: Matching; Assignment Game; Indivisible Goods; Competitive Equilibrium; Lattice.

\section{Introduction}

We study competitive equilibria of markets with indivisible goods. The multiple-partners assignment game with heterogeneous sales and multi-unit demands (a market) is a manyto-many assignment problem with transferable utility in which agents can be partitioned into two disjoint sets: the set of buyers and the set of sellers. In this market sellers deliver indivisible units of (potentially different) goods to buyers who pay a given amount of money for every unit of each good. Each seller owns a given number of indivisible units of each good and each buyer may buy different units of the goods up to an exogenously fixed number which comes from constraints on his capacity for transport, storage, etc. Each seller assigns a per-unit value (or reservation price) to each of the different goods that he owns. Each buyer assigns a valuation (or maximal willingness to pay) to each unit of the different goods; this means that his marginal utility of each of the goods is constant.

There are many assignment problems with these characteristics. ${ }^{1}$ They are many-tomany because each agent can be assigned to (i.e., perform a transaction with) many agents of the other side of the market. They have transferable utility because money may be used as a means of exchange. They are heterogeneous sales because a unit of a particular good owned by a seller may be different from a unit of another good owned by the same seller. They are multi-demanded because buyers may be willing to buy several units of different

\footnotetext{
${ }^{1}$ For instance, the primary market of blood, local markets of fresh products that operate once or twice per week or a clothes market in a city with wholesalers and retailers.
} 
goods. The main questions to be answered are the following. Given an initial distribution of units of the goods among all sellers, (i) what is their optimal assignment to buyers? (ii) what are the prices (if any) that would clear the market?, (iii) what is the subset of goods that are indeed exchanged?, and (iv) what is the set of (overall) utilities that agents might receive?

Given a market, an assignment is a description of how many units of each of the goods are exchanged between every pair formed by a buyer and a seller. An assignment is feasible if it satisfies the quantity and capacity constraints of all agents. A feasible assignment is optimal if it maximizes the total net value (the sum of the valuations minus the reserve price of all exchanged units). It turns out that the set of optimal assignments of a market can be identified with the set of integer solutions of a natural Primal Linear Problem where the objective function (to be maximized) is the total net value, which depends linearly on the assignment, subject to non-negativity constraints and to feasibility constraints. Results on integer programming (see Schrijver, 1996) guarantee that the Primal Linear Program has at least one solution with integer components, since the set of all real-valued solutions of the Primal Linear Program is a polytope whose vertices have all integer-valued coordinates.

To choose an optimal assignment requires information about valuations, reservation prices, and quantity and capacity constraints. Hence, competitive markets may emerge (or be used) as a way of selecting an optimal assignment with low informational requirements. We will assume that buyers and sellers exchange units of the goods with money through competitive markets in which a price vector (a non-negative price for each good) is announced. Given the price vector, each seller determines the optimal number of units he wants to sell of each of the goods he owns and each buyer determines the optimal number of units he wants to buy of each good, without exceeding his capacity constraints. A price vector $p$ is an equilibrium price vector of the market if the plans of all sellers and buyers are compatible at $p$; namely, the market of each good clears in the sense that all optimal plans constitute a feasible and compatible set of exchanges (they are a feasible assignment). In this case we say that the equilibrium price vector and the feasible assignment are compatible. A competitive equilibrium of the market is a pair formed by an equilibrium price vector and a compatible assignment. We show using well-known duality theorems of linear programming that each market has at least a competitive equilibrium. ${ }^{2}$ All our proofs rely

\footnotetext{
${ }^{2}$ In a contemporary and independent paper Milgrom (2009) establishes the existence of competitive equilibrium prices for a more general model which also includes multi-unit auctions and exchange economies as special cases (see Section 2 for a brief description of Milgrom (2009)'s model).
} 
only on well-know results of linear programming. First, we observe that the Dual Linear Problem associated to the Primal Linear Program has a non-empty set of solutions; second, we give a procedure to construct an equilibrium price vector from a given solution of the Dual Linear Program; and third, we show that any optimal solution of the Primal Linear Program is compatible with this equilibrium price vector. Thus, the set of competitive equilibria of a market is intimately related to the set of solutions of the Primal Linear Program (compatible optimal assignments) and the Dual Linear Program (equilibrium price vectors) ${ }^{3}$

We next show that the set of competitive equilibria of a market has a Cartesian product structure: each equilibrium price vector is compatible with all optimal assignments and each optimal assignment is compatible with all equilibrium price vectors. Moreover, the set of equilibrium price vectors has a lattice structure with the natural order of vectors $\geq$ (a reflexive, transitive, antisymmetric, and incomplete binary relation) on the $n$-dimensional Euclidian space, where $n$ is the number of goods and given two vectors $x, y \in \mathbb{R}^{n}, x \geq y$ if and only if $x_{j} \geq y_{j}$ for all $j=1, \ldots, n$. As a consequence of this lattice structure, the set of equilibrium price vectors contains two extreme elements: the sellers-optimal equilibrium price vector with each component being larger or equal to the corresponding component of all other equilibrium price vectors and the buyers-optimal equilibrium price vector with each component being smaller or equal to the corresponding component of all other equilibrium price vectors. We observe that, in contrast to the Shapley and Shubik (1972)'s assignment game, this natural order $\geq$ does not translate into the set of utilities of buyers (nor the set of utilities of sellers) that can be attainable at equilibrium. Partly, this is because there is a insubstantial multiplicity of equilibrium prices of the goods that are not interchanged in any equilibrium assignment. We solve this multiplicity by defining the set of restricted equilibrium price vectors as those equilibrium price vectors for which the price of the goods that are never interchanged in equilibrium is equal to their maximal one without altering the equilibrium property of the full price vector. We show that the set of restricted equilibrium price vectors has a complete lattice structure with the natural order of vectors $\geq$. Then, we show that the set of utilities of buyers that are attainable at equilibrium embeds the lattice structure of the set of restricted equilibrium price vectors. However, we also show that the set of utilities of the sellers that are attainable at equilibrium does not inherit this

\footnotetext{
${ }^{3}$ Shapley and Shubik (1972) already pointed out the relationships among the set of competitive equilibria of a one-to-one assignment game, the core of its associated TU-game, and the solutions of the corresponding primal and dual linear problems.
} 
structure.

There are several papers that have studied generalized versions of Shapley and Shubik (1972)'s one-to-one assignment game to many-to-one or many-to-many models. Camiña (2006), Sotomayor (1999, 2002, 2003, 2007, and 2009) and Milgrom (2009) are some of them. However, part of the emphasis of this literature has been put on the study of alternative cooperative solutions of the associated TU-game, although Camiña (2006), Sotomayor (2007 and 2009) and Milgrom (2009) also study the competitive equilibria of their generalized assignment games. At the end of Section 2 and in Subsection 5.1 we describe this very related literature as well as its connections with our model and results.

Our main contribution to this literature is partially methodological because all our results are proved exclusively through the following well-known linear programming results: the primal and dual linear programs have solutions (integer-valued for the case of the primal), the strong duality theorem and the complementary slackness theorem. Beside of giving an unified treatment this approach has an important computational advantage since there exist several algorithms to compute solutions of linear programs and hence, they can be used to compute competitive equilibria and their associated vectors of agents' utilities. Most of the results in related models extending Shapley and Shubik (1972)'s assignment game use in their proofs existential (and hence, non-computable) arguments. For instance, in Sotomayor (2007) the existence of equilibrium price vectors and its lattice structure (as well as the dual lattice structure of the sets of equilibrium agents' utilities) are obtained by applying Tarski (1955)'s fixed point theorem. Milgrom (2009) proves that the set of equilibrium price vectors has a lattice structure by applying Topkis (1978)'s fixed point theorem.

The paper is organized as follows. In Section 2 we define the multiple-partners assignment game with heterogeneous sales and multi-unit demands (a market) and compare our model with existing related models in the literature. In Section 3, we define optimal assignments and the associated Primal Linear Program of a market. In Section 4 we present the notion of a competitive equilibrium and show its existence by using duality theorems of Linear Programming. In Section 5 we study the structure of the set of competitive equilibria by showing that it is a Cartesian product of the set of equilibrium price vectors times the set of optimal assignments, and that the set of restricted equilibrium price vectors has a complete lattice structure with the natural partial order $\geq$; we also show how this partial order endows a lattice structure to the set of utilities of the buyers (but not to the set of utilities of the sellers) that are attainable at equilibrium. In Section 6 we conclude with 
some final remarks. Appendix 1 at the end of the paper collects omitted proofs. Appendices 2 and 3 at the end of the paper contain some technical and omitted material.

\section{Preliminaries and Related Models}

The multiple-partners assignment game with heterogeneous sales and multi-unit demands (a market) consists of seven objects. The first three are three finite and disjoint sets. The set of $m$ buyers $B=\left\{b_{1}, \ldots, b_{m}\right\}$, the set of $n$ type of goods $G=\left\{g_{1}, \ldots, g_{n}\right\}$, and the set of $t$ sellers $S=\left\{s_{1}, \ldots, s_{t}\right\}$. We identify buyer $b_{i}$ with $i$, good $g_{j}$ with $j$, and seller $s_{k}$ with $k$.

For each buyer $i \in B$ and each good $j \in G$, let $v_{i j} \geq 0$ be the monetary valuation that buyer $i$ assigns to each unit of good $j$; namely, $v_{i j}$ is the maximum price that buyer $i$ is willing to pay for each unit of good $j$. We denote by $V=\left(v_{i j}\right)_{(i, j) \in B \times G}$ the matrix of valuations. Each buyer $i \in B$ can buy at most $d_{i}>0$ units in total. We are assuming that buyers have a constant marginal valuation of each unit of each good and that they are constrained on their total demand. The amount $d_{i}$ should be interpreted as a capacity constraint of buyer $i$ due to limits on his ability for transport, storage, etc. We denote by $d=\left(d_{i}\right)_{i \in B}$ the vector of maximal demands.

For each good $j \in G$ and each seller $k \in S$, let $r_{j k} \geq 0$ be the monetary valuation that seller $k$ assigns to each unit of good $j$; namely, $r_{j k}$ is the reservation (or minimum) price that seller $k$ is willing to accept for each unit of good $j$. We denote by $R=\left(r_{j k}\right)_{(j, k) \in G \times S}$ the matrix of reservation prices. Each seller $k \in S$ has a given number $q_{j k} \in \mathbb{Z}_{+}$of indivisible units of each good $j \in G$, where $\mathbb{Z}_{+}$is the set of non-negative integers. We denote by $Q=\left(q_{j k}\right)_{(j, k) \in G \times S}$ the capacity matrix. Observe that we are admitting the possibility that seller $k$ may have zero units of some of the goods. However, we require that the reservation price for buyer $k$ of a good that he has no units to sell has to be equal to zero; namely, for all $k \in S$ and all $j \in G$,

$$
\text { if } q_{j k}=0 \text { then } r_{j k}=0 .
$$

Moreover, we assume that there is a strictly amount of each good; namely,

$$
\text { for each } j \in G \text { there exists } k \in S \text { such that } q_{j k}>0 \text {. }
$$

A market $M$ is a 7-tuple $(B, G, S, V, d, R, Q)$ satisfying (1) and (2). This constitutes a many-to-many generalization of Shapley and Shubik (1972)'s (one-to-one) assignment game in which each buyer only wants to buy at most one unit (i.e., $d_{i}=1$ for all $i \in B$ ), there is 
only one unit of each good and the set of goods and sellers can be uniquely identified with each other because each seller only owns the unique available unit of a good (i.e., $n=t$ and for all $(j, k) \in G \times S, q_{j k}=1$ if $j=k$ and $q_{j k}=0$ if $\left.j \neq k\right)$. There are other papers that have already extended Shapley and Shubik (1972) model. Camiña (2006) studies an instance of our model in which there is a unique seller that owns $n$ different indivisible objects and each buyer wants to buy at most one object (i.e., $t=1, q_{j 1}=1$ for all $j=1, \ldots, n$, and $d_{i}=1$ for all $i \in B$ ). Sotomayor (2007) studies another extension of the assignment game in which buyers may want to buy several goods although they are not interested in acquiring more than one unit from a given seller, and each seller owns a number of identical and indivisible objects; thus, we can also identify uniquely goods with sellers (i.e., $n=t, d_{i} \in \mathbb{Z}_{+} \backslash\{0\}$ for all $i \in B, q_{j k} \in \mathbb{Z}_{+} \backslash\{0\}$ if $j=k$ and $q_{j k}=0$ if $j \neq k$, and each buyer $i$ and each seller $k$ can exchange at most one unit of the good $j=k$ owned by the seller). ${ }^{4}$

To illustrate why our model is not a particular instance of the models studied by Camiña (2006) and by Sotomayor (2007) consider the market $M=(B, S, G, V, d, R, Q)$, where $B=\left\{b_{1}, b_{2}\right\}, S=\left\{s_{1}, s_{2}\right\}, G=\left\{g_{1}, g_{2}, g_{3}\right\}, d=(20,15)$,

$V=\left(\begin{array}{lll}30 & 15 & 7 \\ 10 & 20 & 6\end{array}\right), R=\left(\begin{array}{ll}5 & 4 \\ 3 & 6 \\ 4 & 5\end{array}\right)$, and $Q=\left(\begin{array}{cc}10 & 8 \\ 7 & 9 \\ 5 & 4\end{array}\right)$.

Observe that (i) there are two sellers and buyers 1 and 2 may buy up to 25 and 15 units respectively, and (ii) there are more goods than sellers and each seller owns several units of the three goods. Thus, by (i) and (ii) our results can not follow from either results in Camiña (2006) or Sotomayor (2007), respectively.

Milgrom (2009) introduces and studies the space of assignment messages to investigate (and solve) the difficulty that agents face, in some mechanism design settings, when reporting their "types" (or valuations of goods, or sets of goods). The model is very general and contains as particular cases multi-unit auctions (with substitutable goods), exchange economies, and integer assignment games. The last one generalizes the Shapley and Shubik (1972)'s model in many ways; in particular, agents (i) may buy some good and sell others (there are no a priori sets of buyers and sellers) and (ii) may trade many units of each good, instead of just one unit. For our model, which is a particular instance of Milgrom (2009), we obtain additional results; for instance, that the set of competitive equilibria is

\footnotetext{
${ }^{4}$ This last condition on the binary nature of the admissible exchanges between a buyer and a seller is not imposed as a restriction on the data of the problem but rather on the set of feasible assignments.
} 
the Cartesian product of the set of equilibrium price vectors and optimal assignments and that the sets of agents' utilities that are attainable at equilibrium partly inherit the lattice structure of the set of equilibrium price vectors.

\section{Optimal Assignments}

In this section we define optimal assignments of a market and show using Linear Programming that they do exist.

An assignment for market $M$ is a matrix $A=\left(A_{i j k}\right)_{(i, j, k) \in B \times G \times S} \in \mathbb{Z}_{+}^{m \times n \times t}$. Given an assignment $A$, each $A_{i j k}$ should be interpreted as follows: buyer $i$ receives $A_{i j k}$ units of good $j$ from seller $k$. When no confusion can arise, we omit the sets to which the subscripts belong to and write, for instance, $\sum_{i j k} A_{i j k}$ and $\sum_{i} A_{i j k}$ instead of $\sum_{(i, j, k) \in B \times G \times S} A_{i j k}$ and $\sum_{i \in B} A_{i j k}$, respectively. We are only interested on assignments satisfying all demand and supply restrictions of feasibility.

Definition 1 The assignment $A$ is feasible for market $M$ if the following two sets of inequalities hold:

(Demand Feasibility) For all $i \in B, \sum_{j k} A_{i j k} \leq d_{i}$.

(Supply Feasibility) For all $(j, k) \in G \times S, \sum_{i} A_{i j k} \leq q_{j k}$.

The inequality in (Demand Feasibility) says that each buyer $i$ buys, in total, at most $d_{i}$ units of all goods while the inequality in (Supply Feasibility) says that each seller $k$ sells at most $q_{j k}$ units of each good $j$. We denote by $F$ the set of all feasible assignments of market $M$.

For each $(i, j, k) \in B \times G \times S$, let

$$
\tau_{i j k}= \begin{cases}v_{i j}-r_{j k} & \text { if } q_{j k}>0 \\ 0 & \text { if } q_{j k}=0\end{cases}
$$

be the per unit gain from the trade of good $j$ between buyer $i$ and seller $k$; observe that if seller $k$ does not have any unit of good $j$ the per unit gain from trade of good $j$ with all buyers is equal to zero and that $\tau_{i j k}$ is negative if $v_{i j}<r_{j k}$. Let $M$ be a market and $A \in F$ be a feasible assignment. We define the total gain from trade of market $M$ at assignment $A$ as

$$
T(A)=\sum_{i j k} \tau_{i j k} \cdot A_{i j k}
$$


Definition 2 A feasible assignment $A^{*}$ is optimal for market $M$ if $T\left(A^{*}\right) \geq T(A)$ holds for any feasible assignment $A \in F$.

We denote by $F^{*}$ the set of all optimal assignments for market $M$.

In order to find the set of optimal assignments for market $M=(B, S, G, V, d, R, Q)$ we consider the following Primal Linear Program (PLP).

Primal linear Program (PLP):

$$
\begin{aligned}
& \max _{\left(A_{i j k}\right)_{(i, j, k) \in B \times G \times S} \in \mathbb{R}^{m \times n \times t}} \sum_{i j k} \tau_{i j k} \cdot A_{i j k} \\
& \text { s. t. (P.1) } \quad \sum_{j k} A_{i j k} \leq d_{i} \quad \text { for all } i \in B \text {, } \\
& \text { (P.2) } \quad \sum_{i} A_{i j k} \leq q_{j k} \text { for all }(j, k) \in G \times S \text {, } \\
& \text { (P.3) } \quad A_{i j k} \geq 0 \quad \text { for all }(i, j, k) \in B \times G \times S \text {. }
\end{aligned}
$$

Results in linear programming guarantee that the set of (real-valued) solutions of the (PLP) is non-empty. Moreover, results in integer programming guarantee that at least one of these solutions has integer components (see Schrijver, 1996); namely, $F^{*} \neq \emptyset$. Appendix 2 at the end of the paper contains a brief description of why the (PLP) has an integer solution. Thus, we state without proof the following result.

Proposition 1 Every market $M$ has a nonempty set of optimal assignments.

\section{Competitive Equilibria}

\subsection{Definition and Existence}

We consider the situation where buyers and sellers trade through competitive markets. That is, there is a unique market (and its corresponding unique price) for each of the goods. Hence, a price vector is an $n$-dimensional vector of non-negative real numbers. Buyers and sellers are price-takers: given a price vector $p=\left(p_{j}\right)_{j \in G} \in \mathbb{R}_{+}^{n}$ sellers supply units of the goods (up to their capacity) in order to maximize revenues at $p$ and buyers demand units of the goods (up to their maximal demands) in order to maximize the total net valuation at $p$.

Supply of seller $k$ For each price vector $p=\left(p_{j}\right)_{j \in G} \in \mathbb{R}_{+}^{n}$, seller $k$ supplies of every good $j$ any feasible amount that maximizes revenues; namely, 


$$
S_{j k}\left(p_{j}\right)= \begin{cases}\left\{q_{j k}\right\} & \text { if } p_{j}>r_{j k} \\ \left\{0,1, \ldots, q_{j k}\right\} & \text { if } p_{j}=r_{j k} \\ \{0\} & \text { if } p_{j}<r_{j k}\end{cases}
$$

To define the demands of buyers we need the following notation. Let $p \in \mathbb{R}_{+}^{n}$ be given and consider buyer $i$. Let

$$
\nabla_{i}^{>}(p)=\left\{j \in G \mid v_{i j}-p_{j}=\max _{j^{\prime} \in G}\left\{v_{i j^{\prime}}-p_{j^{\prime}}\right\}>0\right\}
$$

be the set of goods that give to buyer $i$ the maximum (and strictly positive) net valuation at $p$. Obviously, for some $p$, the set $\nabla_{i}^{>}(p)$ may be empty. Let

$$
\nabla_{i}^{\geq}(p)=\left\{j \in G \mid v_{i j}-p_{j}=\max _{j^{\prime} \in G}\left\{v_{i j^{\prime}}-p_{j^{\prime}}\right\} \geq 0\right\}
$$

be the set of goods that give to buyer $i$ the maximum (and non-negative) net valuation at $p$. Obviously, for some $p$, the set $\nabla_{i}^{\geq}(p)$ may also be empty. Moreover, it is immediate to see that, for all $i \in B$ and all $p \in \mathbb{R}_{+}^{n}$,

$$
\nabla_{i}^{>}(p) \subseteq \nabla_{i}^{\geq}(p)
$$

Demand of buyer $i \quad$ For each price vector $p=\left(p_{j}\right)_{j \in G} \in \mathbb{R}_{+}^{n}$ buyer $i$ demands any feasible amounts of the goods that maximize the net valuations at $p$; namely,

$$
\begin{aligned}
D_{i}(p)=\left\{\alpha=\left(\alpha_{j k}\right)_{(j, k) \in G \times S} \in \mathbb{Z}^{n \times t} \mid\right. & \text { (D.a) } \forall(j, k) \in G \times S, \alpha_{j k} \geq 0, \\
& \text { (D.b) } \sum_{j k} \alpha_{j k} \leq d_{i}, \\
& \text { (D.c) } \nabla_{i}^{>}(p) \neq \emptyset \Longrightarrow \sum_{j k} \alpha_{j k}=d_{i}, \text { and } \\
& \text { (D.d) } \left.\sum_{k} \alpha_{j k}>0 \Longrightarrow j \in \nabla_{i}^{\geq}(p)\right\} .
\end{aligned}
$$

Thus, $D_{i}(p)$ describes the set of all trades that maximize the net valuation of buyer $i$ at $p$. Observe that the set of trades described by each element in the set $D_{i}(p)$ give the same net valuation to buyer $i$; i.e., $i$ is indifferent among all trade plans specified by each $\alpha \in D_{i}(p)$.

Let $A$ be an assignment and let $i$ be a buyer. We denote by $A(i)=\left(A(i)_{j k}\right)_{(j, k) \in G \times S}$ the element in $\mathbb{Z}_{+}^{n \times t}$ such that, for all $(j, k) \in G \times S, A(i)_{j k}=A_{i j k}$.

Definition 3 A competitive equilibrium of market $M$ is a pair $(p, A) \in \mathbb{R}_{+}^{n} \times F \subseteq \mathbb{R}_{+}^{n} \times$ $\mathbb{Z}_{+}^{m \times n \times t}$ such that:

(E.D) For each buyer $i \in B, A(i) \in D_{i}(p)$. 
(E.S) For each good $j \in G$ and each seller $k \in S, \sum_{i} A_{i j k} \in S_{j k}\left(p_{j}\right)$.

We say that a price vector $p$ and a feasible assignment $A$ are compatible if $(p, A)$ is a competitive equilibrium of market $M$. The vector $p \in \mathbb{R}_{+}^{n}$ is an equilibrium price of market $M$ if there exists $A \in F$ such that $(p, A)$ is a competitive equilibrium of market $M$.

Let $P^{*}$ be the set of equilibrium price vectors of market $M$. Theorem 1 below states that the set $P^{*}$ is always non-empty.

Theorem 1 For every market $M, P^{*} \neq \emptyset$.

Milgrom (2009) proves Theorem 1 for a more general model by showing that equilibrium price vectors are the optimal solutions of a non-linear and continuous function of $p$ restricted to a compact set. ${ }^{5}$ However, in Appendix 1 at the end of the paper we include our proof because it only uses linear programming arguments and it is based on computing an optimal assignment (as one of the integer solutions of the Primal Liner Program (PLP)) and a particular equilibrium price vector in $P^{*}$ associated to one of the solutions of the Dual Linear Program (DLP) that we present below.

\subsection{The Dual Linear Program}

In this subsection we present the Dual Linear Program (DLP) and state two well-known results of linear programming: the Strong Duality Theorem and the Complementary Slackness Theorem. Using these two theorems we will show in Theorem 2 that there exists a strong link between the set of competitive equilibria and the set of solutions of the (PLP) and the (DLP).

Let $M=(B, S, G, V, d, R, Q)$ be a market. Let $\gamma=\left(\gamma_{i}\right)_{i \in B} \in \mathbb{R}^{m}$ be an $m$-dimensional vector and $\pi=\left(\pi_{j k}\right)_{(j, k) \in G \times S} \in \mathbb{R}^{n \times t}$ be a $(n \times t)$-matrix (below we give an interpretation of these two objects).

Dual Linear Program (DLP):

$$
\begin{aligned}
& \min _{(\gamma, \pi) \in \mathbb{R}^{m} \times \mathbb{R}^{n \times t}} \sum_{i} d_{i} \cdot \gamma_{i}+\sum_{j k} q_{j k} \cdot \pi_{j k} \\
& \text { s. t. (D.1) } \gamma_{i}+\pi_{j k} \geq \tau_{i j k} \text { for all }(i, j, k) \in B \times G \times S \text {, } \\
& \text { (D.2) } \quad \gamma_{i} \geq 0 \quad \text { for all } i \in B \text {, } \\
& \text { (D.3) } \quad \pi_{j k} \geq 0 \quad \text { for all }(j, k) \in G \times S \text {. }
\end{aligned}
$$

\footnotetext{
${ }^{5}$ Sotomayor (2007) contains an existential proof of the non-emptyness of the set of equilibrium price vectors for her related model based on Tarski (1955)'s fixed point theorem.
} 
Let $D$ be the set of dual feasible solutions (i.e., the set of vectors $\gamma \in \mathbb{R}^{m}$ and matrices $\pi \in \mathbb{R}^{n \times t}$ that satisfy conditions (D.1), (D.2), and (D.3)), and let $D^{*}$ be the set of solutions of the (DLP). Results in linear programming guarantee that the (DLP) has at least a solution (see Schrijver, 1996); namely, $D^{*} \neq \emptyset$. Moreover, $D^{*}$ is a convex subset of $\mathbb{R}^{m} \times \mathbb{R}^{n \times t}$. Thus, we state the following result.

Proposition 2 For every market $M$ the set of solutions $D^{*}$ of the (DLP) in non-empty and convex.

A dual solution $(\gamma, \pi) \in D^{*}$ can be interpreted as a way of sharing the gains of trade among buyers and sellers associated to a particular competitive equilibrium $(p, A)$. The $i^{\text {th }}$ component of vector $\gamma$ describes the (unique) per unit gain of buyer $i$ of all units that he buys and the $(j, k)^{t h}$ element of matrix $\pi$ describes the (unique) per unit gain of seller $k$ of good $j$. For instance, assume that $(\gamma, \pi) \in D^{*}$ and $(p, A)$ is a competitive equilibrium with $A_{i j k}>0$; i.e., buyer $i$ buys at least one unit of good $j$ to seller $k$. Then, as we will formally show later, $\gamma_{i}=v_{i j}-p_{j}$ and $\pi_{j k}=p_{j}-r_{j k}$. Thus, we can identify (almost uniquely) each dual solution with an equilibrium price, and vice versa. As we will see, this identification is not unique. A first (but insubstantial) reason of why this identification is not unique is as follows. Let $(\gamma, \pi) \in D^{*}$ and assume that $q_{j k}=0$ for some $(j, k) \in G \times S$. Let $\pi_{j k}^{\prime} \geq 0$ be arbitrary. Define $\left(\pi_{-j k}, \pi_{j k}^{\prime}\right)$ as the $(n \times t)$-matrix obtained from $\pi$ after replacing $\pi_{j k}$ by $\pi_{j k}^{\prime}$. Then, $\left(\gamma,\left(\pi_{-j k}, \pi_{j k}^{\prime}\right)\right) \in D^{*}$; that is, the value of the $(j, k)^{t h}$ entry of $\pi$ is irrelevant. Hence, we assume without loss of generality that

$$
\pi_{j k}=0 \text { whenever } q_{j k}=0 .
$$

Under this convention, the following result holds.

Proposition 3 For every market $M$ the set of solutions $D^{*}$ of the (DLP) is a compact subset of $\mathbb{R}^{m} \times \mathbb{R}^{n \times t}$.

Let $M$ be a market and $(\gamma, \pi) \in D$ be a dual feasible solution. We write $T^{d}(\gamma, \pi)$ to denote the value of the objective function of the (DLP) at $(\gamma, \pi)$; that is,

$$
T^{d}(\gamma, \pi)=\sum_{i} d_{i} \cdot \gamma_{i}+\sum_{j k} q_{j k} \cdot \pi_{j k}
$$

The Strong Duality Theorem of Linear Programming (see Dantzig, 1963) applied to our setting says that the values of the objective functions of the two linear problems at any pair of solutions coincide; and viceversa, if at a feasible assignment and at a feasible dual the 
two objective functions coincide then they are solutions of their respective linear problems. Formally,

Strong Duality Theorem Let $M$ be a market and assume $A \in F$ and $(\gamma, \pi) \in D$. Then,

$$
A \in F^{*} \text { and }(\gamma, \pi) \in D^{*} \text { if and only if } T(A)=T^{d}(\gamma, \pi) \text {. }
$$

The Complementary Slackness Theorem of Linear Programming (see Schrijver, 1996) says that if a restriction is not binding then the corresponding variable has to be equal to zero and if a variable is not equal to zero then its corresponding restriction has to be binding. Hence, applied to our setting the Complementary Slackness Theorem says the following.

Complementary Slackness Theorem Let $M$ be a market. Then, for all $A \in F^{*}$ and $(\gamma, \pi) \in D^{*}$, the following properties hold:

(CS.1) For all $(i, j, k) \in B \times G \times S, A_{i j k} \cdot\left(\gamma_{i}+\pi_{j k}-\tau_{i j k}\right)=0$.

(CS.2) For all $i \in B,\left(\sum_{j k} A_{i j k}-d_{i}\right) \cdot \gamma_{i}=0$.

(CS.3) For all $(j, k) \in G \times S,\left(\sum_{i} A_{i j k}-q_{j k}\right) \cdot \pi_{j k}=0$.

\subsection{Competitive Equilibria and Solutions of the Linear Programs}

Theorem 2 below says that the set of competitive equilibria (pairs of equilibrium price vectors and compatible assignments) is strongly related to the set of solutions of the two Linear Programs. In order to state and prove it, we need to relate price vectors with dual solutions.

Define the mappings $\gamma(\cdot): \mathbb{R}_{+}^{n} \rightarrow \mathbb{R}_{+}^{m}$ and $\pi(\cdot): \mathbb{R}_{+}^{n} \rightarrow \mathbb{R}_{+}^{n \times t}$ as follows. Let $p \in \mathbb{R}_{+}^{n}$ be given. For each $i \in B$, define

$$
\gamma_{i}(p)= \begin{cases}v_{i j}-p_{j} & \text { if there exists } j \in \nabla_{i}^{>}(p) \\ 0 & \text { otherwise, }\end{cases}
$$

and for each $(j, k) \in G \times S$, define

$$
\pi_{j k}(p)= \begin{cases}p_{j}-r_{j k} & \text { if } p_{j}-r_{j k}>0 \\ 0 & \text { otherwise. }\end{cases}
$$

The number $\gamma_{i}(p)$ is the gain obtained by buyer $i$ from each unit that he wants to buy at $p$ (if any) and the number $\pi_{j k}(p)$ is the profit obtained by seller $k$ from each unit of good $j$ that he wants to sell at $p$ (if any). 
Theorem 2 Let $M$ be a market and let $p \in \mathbb{R}_{+}^{n}$ be a price vector. The following two statements hold.

(2.1) Assume $p \in P^{*}$. Then, $A \in F^{*}$ if and only if $p$ and $A$ are compatible.

(2.2) $p \in P^{*}$ if and only if $(\gamma(p), \pi(p)) \in D^{*}$.

Proof See Appendix 1 at the end of the paper.

Corollary 1 The set of equilibrium price vectors $P^{*}$ is a convex and compact subset of $\mathbb{R}_{+}^{n}$.

\section{Structure of the Set of Competitive Equilibria}

\subsection{Previous results}

Recall that the assignment game of Shapley and Shubik (1972) is a particular instance of our model where each seller owns one indivisible object and each buyer wants to buy at most one object. Since objects owned by different sellers may be perceived differently by different buyers (or they may, indeed, be different), we can identify the set of goods $G$ with the set of sellers $S$. Namely, a market $M$ is an assignment game if $d_{i}=1$ for all $i \in B$, $n=t$ and for all $(j, k) \in G \times S$,

$$
q_{j k}= \begin{cases}1 & \text { if } j=k \\ 0 & \text { if } j \neq k\end{cases}
$$

Hence, each seller $j \in S$ has a reservation value $r_{j} \geq 0$ of the indivisible object $j \in G$ that he owns. Thus, an assignment game can be identified as an $(m \times t)$-matrix $a$, where for all $(i, j) \in B \times S, a_{i j}=\max \left\{0, v_{i j}-r_{j}\right\}$.

The set of competitive equilibria of a (one-to-one) assignment game has the following four properties.

(1) The set of equilibrium price vectors is a non-empty, convex and compact subset of $\mathbb{R}_{+}^{n}$.

(2) The set of competitive equilibria is the Cartesian product of the set of equilibrium price vectors times the set of optimal assignments.

(3) The set of equilibrium price vectors $P^{*}$ endowed with the partial order $\geq$ on $\mathbb{R}_{+}^{n}$ (where $p \geq p^{\prime}$ if and only if $p_{j} \geq p_{j}^{\prime}$ for all $j \in G$ ) is a complete lattice. ${ }^{6}$ In particular,

\footnotetext{
${ }^{6}$ See Appendix 3 for a self-contained definition of a complete lattice.
} 
given $p, p^{\prime} \in P^{*},\left(\max \left\{p_{j}, p_{j}^{\prime}\right\}\right)_{j \in G} \in P^{*}$ and $\left(\min \left\{p_{j}, p_{j}^{\prime}\right\}\right)_{j \in G} \in P^{*}$. Moreover, the set of equilibrium price vectors contains two extreme vectors $p^{B}$ and $p^{S}$ with the property that for any equilibrium price vector $p \in P^{*}, p^{S} \geq p \geq p^{B}$.

(4) The lattice structure of $P^{*}$ is translated into the set of utilities that are attainable at equilibrium as follows. Given $p \in P^{*}$ and an optimal assignment $\mu=\left(\mu_{i j}\right)_{(i, j) \in B \times S}$, define for each $i \in B$,

$$
u_{i}(p)= \begin{cases}v_{i j}-p_{j} & \text { if } \mu_{i j}=1 \text { for some } j \in S \\ 0 & \text { otherwise }\end{cases}
$$

and for each $j \in S$,

$$
w_{j}(p)= \begin{cases}p_{j}-r_{j} & \text { if } \mu_{i j}=1 \text { for some } i \in B \\ 0 & \text { otherwise. }\end{cases}
$$

It turns out that these utilities are independent of the chosen optimal assignment $\mu$ (see Lemma 6 below for a proof of this statement in our more general many-to-many setting). Thus, we can write them as depending only on the equilibrium price vector $p$. Then, for all $p, p^{\prime} \in P^{*}$, the following three statements are equivalent:

(a) $p_{j} \geq p_{j}^{\prime}$ for all $j \in G$.

(b) $u_{i}\left(p^{\prime}\right) \geq u_{i}(p)$ for all $i \in B$.

(c) $w_{j}(p) \geq w_{j}\left(p^{\prime}\right)$ for all $j \in S$.

Hence, we can define two binary relations $\succeq_{u}$ and $\succeq_{w}$ on $P^{*}$ as follows: for $p, p^{\prime} \in P^{*}$,

$$
p \succeq_{u} p^{\prime} \Longleftrightarrow u_{i}(p) \geq u_{i}\left(p^{\prime}\right) \text { for all } i \in B,
$$

and

$$
p \succeq_{w} p^{\prime} \Longleftrightarrow w_{j}(p) \geq w_{j}\left(p^{\prime}\right) \text { for all } j \in S .
$$

Then, the set $P^{*}$ endowed with the partial order $\succeq_{u}$ (or $\left.\succeq_{w}\right)$ is a complete lattice. Moreover, $\succeq_{u}$ and $\succeq_{w}$ are dual in the sense that $p \succeq_{u} p^{\prime} \Longleftrightarrow p^{\prime} \succeq_{w} p$.

Consider again our model. We have already seen (in Theorem 1 and Corollary 1) that property (1) still holds while Milgrom (2009) shows using Topkis (1978)'s theorem that property (3) also holds. In this section we will show that property (2) is satisfied while property (4) only holds partially. In particular, the equivalences between the statements (a), (b), and (c) above do not hold anymore on $P^{*}$. One of the reasons is because there may be goods that are never exchanged in equilibrium; for instance, because the smallest reserve price $\underline{r}_{j}=\min _{k \in S} r_{j k}$ of good $j$ is strictly larger than its largest valuation $\bar{v}_{j}=\max _{i \in B} v_{i j}$. 
Then, the price of good $j$ can be equal to any number in the interval $\left[\bar{v}_{j}, \underline{r}_{j}\right]$ without affecting the equilibrium property of the full vector. We shrink the set of equilibrium price vectors by fixing the price of the goods that are never exchanged at equilibrium at the highest possible one that keeps the equilibrium properties of the full price vector. We call it set the set of restricted equilibrium price vectors and denote it by $P^{* *}$. Then, we show in Theorem 3 that $P^{* *}$ has also a complete lattice structure with the natural order $\geq$ of vectors. ${ }^{7}$ Moreover, we show that the equivalence of (a) and (b) above holds on $P^{* *}$ and that property (c) above is not anymore equivalent to properties (a) and (b) on the set $P^{* *}$; i.e., for all $p, p^{\prime} \in P^{* *}$, (a) and (b) are equivalent and each implies (c) but (c) neither implies (a) nor (b).

Before proceeding we compare these results with similar results obtained in related models. Camiña (2006) shows that in her model with one seller and unit-demands the set of core utilities has the following properties: (i) it is non-empty, (ii) it may not coincide with the set of utilities that are attainable at equilibrium, and (iii) it forms a complete lattice. In Sotomayor (2007)'s model where there are the same number of goods and sellers, each seller only owns (potentially many) units of one good and exchanges are binary (i.e., $A_{i j k} \in\{0,1\}$ for all $\left.(i, j, k) \in B \times G \times S\right)$ it is showed that the sets of agents' utilities attainable at equilibrium have a dual lattice structure with the partial order $\geq$ on $\mathbb{R}^{n}$. This is inherited from the lattice of the set of equilibrium price vectors because each seller only owns units of one good. Moreover, Sotomayor (2007)'s proof applies Tarski (1955)'s algebraic fixed point theorem to an order-preserving and non-identical map whose fixed points are the set of utilities that are attainable at equilibrium. In contrast, our results are different and are exclusively based on duality theorems of linear programming. Sotomayor (2009) extends her previous results to a more general model in which any two agents from each side of the market may form a partnership, contribute with an (identical) amount of labor (that may be perfectly divisible) and generate an amount of income which has to be divided among the two of them. Sotomayor (2009) shows the relationships that hold among several solution concepts and their non-emptyness. Finally, Milgrom (2009) does not address properties (2) and (4), and complements property (3) by showing, using Topkis (1978)'s theorem, that in his general setting the set of market-clearing prices is a non-empty, closed, and convex sublattice (a subset of a lattice that is itself a lattice).

The main methodological contribution of our paper is that all our results on the gen-

\footnotetext{
${ }^{7}$ Our proof is direct and it does not use Topkis (1978)'s theorem. In addition, with a few slight modifications it can be adapted to prove directly that $P^{*}$ has a complete lattice structure with the order $\geq$.
} 
eralized many-to-many assignment game are exclusively based on linear programming arguments: (i) the identification of optimal assignments and competitive equilibrium prices with the solutions of the primal and dual linear programs, respectively; and (ii) the Strong Duality and the Complementary Slackness Theorems. As we have already argued in the Introduction, this approach is useful because we can obtain optimal assignments and equilibrium price vectors by means of any of the many existing algorithms that compute solutions of linear programs.

\subsection{Cartesian Product Structure of the Set of Competitive Equi- libria}

We first establish that in our model the set of competitive equilibria has a Cartesian product structure; namely, if $(p, A)$ and $\left(p^{\prime}, A^{\prime}\right)$ are two competitive equilibria of $M$ then, $\left(p, A^{\prime}\right)$ and $\left(p^{\prime}, A\right)$ are also two competitive equilibria of $M$. This follows immediately from Lemmata 2 and 4 used to prove Theorem 2. We state it as Proposition 4 below.

Proposition 4 Let $M$ be a market. Then, $(p, A)$ is a competitive equilibrium of $M$ if and only if $p \in P^{*}$ and $A \in F^{*}$.

Proof Assume $(p, A)$ is a competitive equilibrium of $M$. By definition, $p \in P^{*}$. Moreover, $p$ and $A$ are compatible. By Lemma 2, $A \in F^{*}$. Assume $p \in P^{*}$ and $A \in F^{*}$. By Lemma 4, $p$ and $A$ are compatible. Thus, $(p, A)$ is a competitive equilibrium of $M$.

\subsection{Sets of Equilibrium Utilities}

Let $p \in \mathbb{R}_{+}^{n}$ be a price vector and $A \in F$ a feasible assignment of market $M$. We define the utility of buyer $i \in B$ at the pair $(p, A)$ as the total net gain obtained by $i$ from his exchanges specified by $A$ at price $p$. We denote it by $u_{i}(p, A)$; namely,

$$
u_{i}(p, A)=\sum_{j k}\left(v_{i j}-p_{j}\right) \cdot A_{i j k} .
$$

We define the utility of seller $k \in S$ at the pair $(p, A)$ as the total net gain obtained by $k$ from his exchanges specified by $A$ at price $p$. We denote it by $w_{k}(p, A)$; namely,

$$
w_{k}(p, A)=\sum_{i j}\left(p_{j}-r_{j k}\right) \cdot A_{i j k} .
$$

Define

$$
G^{>}=\left\{j \in G \mid \text { there exists } A \in F^{*} \text { such that } A_{i j k}>0 \text { for some }(i, k) \in B \times S\right\}
$$


as the set of goods that are exchanged at some optimal assignment. For each seller $k \in S$, define

$$
G_{k}^{>}=\left\{j \in G \mid \text { there exists } A \in F^{*} \text { such that } A_{i j k}>0 \text { for some } i \in B\right\}
$$

as the set of goods of which $k$ sells strictly positive amounts at some optimal assignment. Obviously, $G^{>}=\cup_{k \in S} G_{k}^{>}$.

Next lemma states that at equilibrium utilities are independent of the particular optimal assignment chosen since they only depend on the equilibrium price vector (which determines the associated solution of the (DLP)).

Lemma 6 Let $p \in P^{*}$ be an equilibrium price vector of $M$ and let $A \in F^{*}$ be an optimal assignment of $M$. Then, the following two conditions hold:

(L6.1) For each buyer $i \in B, u_{i}(p, A)=\gamma_{i}(p) \cdot d_{i}$.

(L6.2) For each seller $k \in S, w_{k}(p, A)=\sum_{j \in G_{k}^{>}}\left(p_{j}-r_{j k}\right) \cdot q_{j k}{ }^{8}$

Proof of Lemma 6 Let $(p, A) \in P^{*} \times F^{*}$. Note that $p$ and $A$ are compatible. To prove (L6.1), fix $i \in B$. By definition, $u_{i}(p, A)=\sum_{j k}\left(v_{i j}-p_{j}\right) \cdot A_{i j k}$. Let $(j, k) \in G \times S$ be given. If $A_{i j k}=0$ then, $\left(v_{i j}-p_{j}\right) \cdot A_{i j k}$ can trivially be written as $\gamma_{i}(p) \cdot A_{i j k}$. If $A_{i j k} \neq 0$ then, by condition (D.d) in the definition of $D_{i}(p), j \in \nabla_{i}^{\geq}(p)$, which implies that $\left(v_{i j}-p_{j}\right)=\gamma_{i}(p)$, and

$$
u_{i}(p, A)=\gamma_{i}(p) \cdot\left(\sum_{j k} A_{i j k}\right) .
$$

If $\gamma_{i}(p)=0$ then, the statement holds because $\gamma_{i}(p) \cdot\left(\sum_{j k} A_{i j k}\right)=\gamma_{i}(p) \cdot d_{i}=0$. By condition (CS.2) in the Complementary Slackness Theorem, if $\gamma_{i}(p) \neq 0$ then $\sum_{j k} A_{i j k}=d_{i}$. Thus,

$$
u_{i}(p, A)=\gamma_{i}(p) \cdot d_{i}
$$

To prove (L6.2), fix $k \in S$. By definition, $w_{k}(p, A)=\sum_{i j}\left(p_{j}-r_{j k}\right) \cdot A_{i j k}$. Then,

$$
\sum_{i j}\left(p_{j}-r_{j k}\right) \cdot A_{i j k}=\sum_{j}\left(p_{j}-r_{j k}\right) \cdot\left(\sum_{i} A_{i j k}\right) .
$$

Since $p \in P^{*}$, by (E.S), if $\left(p_{j}-r_{j k}\right)>0$ then $\sum_{i} A_{i j k}=q_{j k}$. If $\left(p_{j}-r_{j k}\right)<0$ then, $S_{j k}\left(p_{j}\right)=\{0\}$. Hence, since $p$ and $A$ are compatible, $\sum_{i} A_{i j k}=0$. Therefore,

$$
w_{k}(p, A)=\sum_{j \in\left\{j^{\prime} \in G \mid p_{j^{\prime}}-r_{j^{\prime} k} \geq 0\right\}}\left(p_{j}-r_{j k}\right) \cdot q_{j k}=\sum_{j \in G_{k}^{>}}\left(p_{j}-r_{j k}\right) \cdot q_{j k} .
$$

\footnotetext{
${ }^{8}$ Observe that $w_{k}(p, A)$ can also be written as $\sum_{j \in G} \pi_{j k}(p) \cdot q_{j k}$.
} 
Condition (12) holds because $\left\{j^{\prime} \in G \mid q_{j^{\prime} k}>0\right.$ and $\left.p_{j^{\prime}}-r_{j^{\prime} k}>0\right\} \subseteq G_{k}^{>} \subseteq\{j \in G \mid$ $\left.p_{j}-r_{j k} \geq 0\right\}$. To see that, let $j \in G_{k}^{>}$. Hence, there exists $\bar{A} \in F^{*}$ such that $\bar{A}_{i j k}>0$, which implies, since $p$ and $\bar{A}$ are compatible, $p_{j}-r_{j k} \geq 0$. Thus, the second inclusion holds. To prove the first one, assume $j \in\left\{j^{\prime} \in G \mid q_{j^{\prime} k}>0\right.$ and $\left.p_{j^{\prime}}-r_{j^{\prime} k}>0\right\}$. Then, since $p \in P^{*}$, by (E.S), $\sum_{i} A_{i j k}=q_{j k}$. Thus, $j \in G_{k}^{>}$.

By Lemma 6, we can write the utilities of buyers and sellers as functions only of the equilibrium price vector $p$; namely, given $p \in P^{*}$, we write for each $i \in B$ and each $k \in S$,

$$
u_{i}(p)=\gamma_{i}(p) \cdot d_{i}
$$

and

$$
w_{k}(p)=\sum_{j \in G_{k}^{>}}\left(p_{j}-r_{j k}\right) \cdot q_{j k}
$$

\subsection{The Set of Restricted Equilibrium Price Vectors}

We start this subsection with an example that illustrates two important facts. First, it shows that, in contrast with the Shapley and Shubik (1972)'s assignment game and Sotomayor (2007)'s generalization, there are markets with two equilibrium price vectors $p, p^{\prime} \in P^{*}$ with the property that $w_{k}\left(p^{\prime}\right)>w_{k}(p)$ for all $k \in S$ while $u_{i}\left(p^{\prime}\right)>u_{i}(p)$ for some $i \in B$ (the equivalence between statements (b) and (c) at the beginning of Section 5 does not hold on $\left.P^{*}\right)$. Second, it also shows that the (incomplete) binary relation $\geq$ on the set of vectors in $\mathbb{R}_{+}^{n}$ is not imbedded into the set of attainable equilibrium utilities (the equivalence between statements (a) and (b) at the beginning of Section 5 does not hold on $P^{*}$ ). These two facts will have consequences for the lattice structures of the set(s) of (restricted) equilibrium price vectors and the sets of attainable equilibrium utilities that will be analyzed at the end of this subsection.

Example 1 Let $M=(B, G, S, V, d, R, Q)$ be a market where $B=\left\{b_{1}, b_{2}\right\}, G=\left\{g_{1}, g_{2}, g_{3}\right\}$,

$S=\left\{s_{1}\right\}, V=\left(\begin{array}{lll}8 & 0 & 2 \\ 0 & 5 & 3\end{array}\right), d=(2,3), R=\left(\begin{array}{c}1 \\ 2 \\ 10\end{array}\right)$, and $Q=\left(\begin{array}{c}2 \\ 3 \\ 1\end{array}\right)$. It is easy to see

that, for all $p_{3}, p_{3}^{\prime} \in[3,10], p=\left(5,4, p_{3}\right)$ and $p^{\prime}=\left(7,2, p_{3}^{\prime}\right)$ are two equilibrium price vectors of $M$ and $14=w_{1}(p)>w_{1}\left(p^{\prime}\right)=12$. Furthermore, $\gamma(p)=(3,1)$ and $\gamma\left(p^{\prime}\right)=(1,3)$. Then, $u_{1}(p)=3 \cdot 2=6, u_{2}(p)=1 \cdot 3=3, u_{1}\left(p^{\prime}\right)=1 \cdot 2=2$, and $u_{2}\left(p^{\prime}\right)=3 \cdot 3=9$. Thus, 
$w_{1}(p)>w_{1}\left(p^{\prime}\right)$ and $u_{1}(p)>u_{1}\left(p^{\prime}\right)$ and $u_{2}\left(p^{\prime}\right)>u_{2}(p)$. Moreover, observe that, for all $i \in$ $\{1,2\}, u_{i}\left(7,2, p_{3}\right)=u_{i}\left(7,2, p_{3}^{\prime}\right)$ for all $3 \leq p_{3}<p_{3}^{\prime} \leq 10$ but $p=\left(7,2, p_{3}\right)<\left(7,2, p_{3}^{\prime}\right)=p^{\prime}$. This is because no unit of good 3 is exchanged in any equilibria and hence, the equilibrium price vector $p=\left(7,2, p_{3}\right)$ is equivalent (in terms of its induced demands and supplies) to the equilibrium price vector $p^{\prime}=\left(7,2, p_{3}^{\prime}\right)$ as long as $3 \leq p_{3}<p_{3}^{\prime} \leq 10$.

In order to restore the interesting property that the (incomplete) binary relation $\geq$ on $\mathbb{R}_{+}^{n}$ reproduces itself in terms of buyers utilities (in the corresponding space) we have to eliminate an insubstantial multiplicity of equilibrium prices of the goods that are not exchanged at any equilibrium assignment. We do it by setting the prices of each nonexchanged good equal to the highest possible one (keeping the equilibrium property of the price vector). ${ }^{9}$ Formally, given an equilibrium price vector $p \in P^{*}$, define $\bar{p}=\left(\bar{p}_{j}\right)_{j \in G}$ as follows:

$$
\bar{p}_{j}= \begin{cases}p_{j} & \text { if } j \in G^{>} \\ p_{j}^{S} & \text { if } j \notin G^{>},\end{cases}
$$

where $p_{j}^{S}=\sup _{p \in P^{*}} p_{j} \cdot{ }^{10}$ Proposition 5 below says that this distortion does not affect the equilibrium property of the original price vector.

Proposition 5 Let $M$ be a market and let $p \in P^{*}$. Then, $\bar{p} \in P^{*}$.

Proof See Appendix 1 at the end of the paper

Proposition 6 shows that the distortion in (15) coincides with the one produced in $p$ by computing its associated price vector $p^{(\gamma(p), \pi(p))}$ from its dual solution $(\gamma(p), \pi(p))$ (see (16) in Appendix 1).

Proposition 6 For every $p \in P^{*}, p^{(\gamma(p), \pi(p))}=\bar{p}$.

Proof See Appendix 1 at the end of the paper.

Given a market $M$, we can define the set of restricted equilibrium price vectors $P^{* *}$ as those that are obtained from equilibrium price vectors after setting the price of the goods

\footnotetext{
${ }^{9}$ The choice of the highest price is arbitrary. The important fact is to select, for each of these goods, just one of its potentially many equilibrium prices.

${ }^{10}$ The vector $p^{S}=\left(p_{j}^{S}\right)_{j \in G}$ is called the sellers-optimal equilibrium price. Similarly, define for each $j \in G$, $p_{j}^{B}=\inf _{p \in P^{*}} p_{j}$. The vector $p^{B}=\left(p_{j}^{B}\right)_{j \in G}$ is called the buyers-optimal equilibrium price. By Milgrom (2009), the price vectors $p^{S}$ and $p^{B}$ do exist and they are the two extreme equilibrium prices of the complete lattice $\left(P^{*}, \geq, \vee, \wedge\right)$.
} 
that are not exchanged at any optimal assignment equal to their sellers-optimal equilibrium price. Namely,

$$
P^{* *}=\left\{p \in P^{*} \mid p_{j}=p_{j}^{S} \text { for every } j \notin G^{>}\right\} .
$$

Theorem 3 below states that the set $P^{* *}$ has a complete lattice structure with the natural order $\geq$ on $\mathbb{R}_{+}^{n} \cdot{ }^{11}$

Theorem 3 The four-tuple $\left(P^{* *}, \geq, \vee, \wedge\right)$ is a complete lattice.

Proof See Appendix 1 at the end of the paper.

Our objective in the remaining of this subsection is to show how the complete lattice structure with the natural order $\geq$ on $\mathbb{R}_{+}^{n}$ (and on $P^{*}$ and $P^{* *}$ ) translates into the set of of agents' utilities that are attainable at equilibrium. The fact that the lattice structure of the set of equilibrium price vectors is inherited in a dual way by the sets of equilibrium utilities of buyers and sellers is an important property because it says that there is a conflict of interests between the two sides of the market (and unanimity in each of the sides) with respect to two comparable equilibrium price vectors.

Define the partial orders $\succeq_{u}$ and $\succeq_{w}$ on $P^{*}$ as follows: for any pair $p, p^{\prime} \in P^{*}$,

$$
p \succeq_{u} p^{\prime} \text { if and only if } u_{i}(p) \geq u_{i}\left(p^{\prime}\right) \text { for every } i \in B
$$

and

$$
p \succeq_{w} p^{\prime} \text { if and only if } w_{k}(p) \geq w_{k}\left(p^{\prime}\right) \text { for every } k \in S \text {. }
$$

Example 1 has showed that we may have $p, p^{\prime} \in P^{*}$ with the property that $p \neq p^{\prime}$, but $u_{i}(p)=u_{i}\left(p^{\prime}\right)$ for all $i \in B$; i.e., the binary relation $\succeq_{u}$ is not a partial order on $P^{*}$ because it is not antisymmetric since $p \succeq_{u} p^{\prime}, p^{\prime} \succeq_{u} p$ and $p \neq p^{\prime}$ hold. Hence, the lattice structure of the set $P^{*}$ with the binary relation $\geq$ is not inherited by the set of utilities of buyers that are attainable at equilibrium. However, next proposition says that the partial order $\geq$ on the set of restricted equilibrium price vectors translates into the set of utilities of the buyers that are attainable at equilibrium (i.e., the statements (a) and (b) at the beginning of Section 5 are equivalent on this subset of $\left.P^{*}\right)$. Formally,

Proposition 7 Let $p, p^{\prime} \in P^{* *}$ be two restricted equilibrium price vectors of market $M$. Then,

$$
u_{i}(p) \geq u_{i}\left(p^{\prime}\right) \text { for every } i \in B \text { if and only if } p_{j}^{\prime} \geq p_{j} \text { for every } j \in G .
$$

\footnotetext{
${ }^{11}$ In order to state our results we present in Appendix 3 some notions to define a lattice in our setting. See Birkoff (1979) for a general description of lattice theory.
} 
Proof See Appendix 1 at the end of the paper.

Consider now the restriction of the partial order $\succeq_{u}$ on the set $P^{* *}$ and define the binary operations $\vee_{u}$ and $\wedge_{u}$ on $P^{* *}$ as the binary operations on $P^{*}$ restricted to the set $P^{* *}$; namely, for all $p, p^{\prime} \in P^{* *}$,

$$
p \vee_{u} p^{\prime} \equiv \check{p} \text { and } p \wedge_{u} p^{\prime} \equiv \hat{p}
$$

Theorem 4 The four-tuple $\left(P^{* *}, \succeq_{u}, \vee_{u}, \wedge_{u}\right)$ is a complete lattice.

Proof It follows from Theorem 3 and Proposition 7.

Next proposition shows that the conflict of interests between the two sides of the market on the set of equilibrium price vectors holds partially in our general model (statement (b) in the beginning of Section 5 implies statement (c) on $P^{*}$ ); namely, if buyers unanimously consider the equilibrium price vector $p$ as being at least as good as equilibrium price vector price $p^{\prime}$ then all sellers consider $p^{\prime}$ as being at least as good as $p$ (remember that Example 1 shows that the converse does not hold).

Proposition 8 Let $p, p^{\prime} \in P^{*}$ be two equilibrium price vectors of market $M$ such that $u_{i}(p) \geq u_{i}\left(p^{\prime}\right)$ for all $i \in B$. Then, $w_{k}\left(p^{\prime}\right) \geq w_{k}(p)$ for all $k \in S$.

Proof Let $p, p^{\prime} \in P^{*}$ and assume that $u_{i}(p) \geq u_{i}\left(p^{\prime}\right)$ for every $i \in B$. By Lemma 8 in Appendix $1, p_{j}^{\prime} \geq p_{j}$ for every $j \in G^{>}$. Fix $k \in S$. Then, $p_{j}^{\prime}-r_{j k} \geq p_{j}-r_{j k}$ for every $j \in G_{k}^{>}$. Thus, by $(14), w_{k}\left(p^{\prime}\right) \geq w_{k}(p)$.

Proposition 9 states that utilities associated to the two extreme equilibrium price vectors $p^{B}$ and $p^{S}$ are extreme and opposite utilities.

Proposition 9 Let $M$ be a market. Then, for every $p \in P^{*}$, the following properties hold.

(9.1) For every $i \in B, u_{i}\left(p^{B}\right) \geq u_{i}(p) \geq u_{i}\left(p^{S}\right)$.

(9.2) For every $k \in S, w_{k}\left(p^{S}\right) \geq w_{k}(p) \geq w_{k}\left(p^{B}\right)$.

Proof Consider any $p \in P^{*}$. By their definitions, $p_{j}^{B} \leq p_{j} \leq p_{j}^{S}$ for all $j \in G$. In particular, these inequalities hold for all $j \in G^{>}$. By Lemma 8 in Appendix $1, u_{i}\left(p^{B}\right) \geq$ $u_{i}(p) \geq u_{i}\left(p^{S}\right)$ for all $i \in B$. Thus, (9.1) holds. By Proposition $8, w_{k}\left(p^{B}\right) \leq w_{k}(p) \leq w_{k}\left(p^{S}\right)$ for all $k \in S$. Thus, (9.2) holds.

Consider again Example 1. Take $p=(3,2,10)$ and $p^{\prime}=\left(\frac{3}{2}, 3,10\right)$ and observe that $p, p^{\prime} \in P^{* *}$ and $w_{1}(p)=w_{1}\left(p^{\prime}\right)=4$. Hence, $p \succeq_{w} p^{\prime}, p^{\prime} \succeq_{w} p$, and $p \neq p^{\prime}$. Thus, the binary 
relation $\succeq_{w}$ is not a partial order on $P^{* *}$ because it is not antisymmetric. Hence, the set $P^{* *}$ does not have a lattice structure with the binary relation $\succeq_{w}$ (and the induced binary operations $\vee_{w}$ and $\wedge_{w}$ ). Observe that this is a direct consequence of the fact that in our model sellers may own units of different goods. Therefore, two unrelated equilibrium price vectors in $P^{* *}$ may give the same utility to a seller because the losses in revenues from selling one good with a lower price are compensated with the gains from selling another good with a higher price. Obviously, this can not occur whenever each seller only owns units of a unique good, as in Sotomayor (2007 and 2009).

\section{Concluding Remarks}

Before finishing the paper some remarks are in order. The first one is related with the computational advantage of the linear programming approach. It allows to find the (essentially) unique optimal assignment $A^{*}$ as the (essentially) unique solution of the (PLP) as well as the set of equilibrium price vectors as solutions of the (DLP). Moreover, and following Leonard (1983), there is an alternative way to compute the two extreme equilibrium price vectors $p^{S}$ and $p^{B}$ as solutions of two linear programs. To find $p^{S}$ we first compute $\left(\gamma^{S}, \pi^{S}\right)$, the best dual solution from the point of view of the sellers, by letting $T \equiv T\left(A^{*}\right)$ and solving the following associated dual program: choose $(\gamma, \pi) \in \mathbb{R}^{m} \times \mathbb{R}^{n \times t}$ in order to

$$
\begin{aligned}
& \max _{(\gamma, \pi) \in \mathbb{R}^{m} \times \mathbb{R}^{n \times t}} \sum_{j k} q_{j k} \cdot \pi_{j k} \\
& \text { s. t. (D.0) } \quad \sum_{i} d_{i} \cdot \gamma_{i}+\sum_{j k} q_{j k} \cdot \pi_{j k}=T \\
& \gamma_{i}+\pi_{j k} \geq \tau_{i j k} \quad \text { for all }(i, j, k) \in B \times G \times S, \\
& \gamma_{i} \geq 0 \quad \text { for all } i \in B \text {, } \\
& \pi_{j k} \geq 0 \quad \text { for all }(j, k) \in G \times S \text {, } \\
& \pi_{j k}=0 \quad \text { if } q_{j k}=0 \text {. }
\end{aligned}
$$

It is easy to show that $\left(\gamma^{S}, \pi^{S}\right)$ is the unique solution of this linear program, which is among all solutions of the (DLP) the one with the highest entries in the matrix $\pi$. Then, set $p^{S}=p^{\left(\gamma^{S}, \pi^{S}\right)}$. Analogously, to find $p^{B}$ we first compute $\left(\gamma^{B}, \pi^{B}\right)$ by solving a symmetric linear program. Then, set $p^{B}=p^{\left(\gamma^{B}, \pi^{B}\right)}$. Thus $p^{S}$ and $p^{B}$ are obtained by solving two dual linear programs.

Second, the classical approach in matching of transforming many-to-many or many-toone ordinal problems into a one-to-one matching problem by breaking each buyer $i$ and each 
seller $k$ into as many pieces as his capacity constraint ( $d_{i}$ and $\sum_{j} q_{j k}$, respectively) would not work here because in the one-to-one setting two units held by two different "pieces" of a seller would have to be considered as different goods and hence, they could have different prices.

Finally, we leave for future research the study of alternative cooperative notions like pairwise stability, core, or set-wise stability of the natural TU-game associated to our market and the analysis of their relationships with the set of competitive equilibria (Camiña (2006) and Sotomayor (2002, 2007, and 2009) perform parts of this analysis in their respective settings). We conjecture that the core and the set-wise stable set are non-empty, they are different sets, and the set of utilities attainable at equilibrium is a strict subset of the set of set-wise stable utilities. We also leave for future research the characterization of (strategic) equilibria when sellers (or buyers) set prices simultaneously and the relationship of these sets with the set of competitive equilibrium prices of a market $M$.

\section{References}

[1] Birkhoff, G. Lattice Theory. American Mathematical Society, Colloquium Publications 25; third edition (1979).

[2] Camiña, E. "A generalized assignment game," Mathematical Social Sciences 52, 152161 (2006).

[3] Dantzig, G. Linear Programming and Extensions. Princeton University Press (1963).

[4] Grätzer, G. General Lattice Theory. Birkhäuser Verlag (2003).

[5] Leonard, H. "Elicitation of honest preferences for the assignment of individuals to positions," Journal of Political Economy 91, 461-479 (1983).

[6] Milgrom, P. "Assignment messages and exchanges," forthcoming American Economic Review: Micro (2009).

[7] Schrijver, A. Theory of Linear and Integer Programming. John Wiley and Sons (1996).

[8] Shapley, L. S., and Shubik, M. "The assignment game I: the core," International Journal of Game Theory 1, 111-130 (1972). 
[9] Sotomayor, M. "The lattice structure of the set of stable outcomes of the multiple partners assignment game," International Journal of Game Theory 28, 567-583 (1999).

[10] Sotomayor, M. "A labor market with heterogeneous firms and workers," International Journal of Game Theory 31, 269-283 (2002).

[11] Sotomayor, M. "Some further remark on the core structure of the assignment game," Mathematical Social Sciences 46, 261-265 (2003).

[12] Sotomayor, M. "Connecting the cooperative and competitive structures of the multiplepartners assignment game," Journal of Economic Theory 134, 155-174 (2007).

[13] Sotomayor, M. "Correlating new cooperative and competitive concepts in the timesharing assignment game," mimeo (2009).

[14] Topkis, D. "Minimizing a submodular function on a lattice," Operations Research 26, 305-321 (1978).

[15] Tarski, A. "A lattice-theoretical fix-point theorem and its applications," Pacific Journal of Mathematics 5, 285-309 (1955). 


\section{Appendix 1: Omitted proofs}

Before proving Theorem 1 we define for each solution $\left(\gamma^{*}, \pi^{*}\right) \in D^{*}$ of the (DLP) its associated price vector $p^{\left(\gamma^{*}, \pi^{*}\right)}=\left(p_{j}^{\left(\gamma^{*}, \pi^{*}\right)}\right)_{j \in G}$ as follows. For each $j \in G$,

$$
p_{j}^{\left(\gamma^{*}, \pi^{*}\right)}=\min _{\left\{k \in S \mid q_{j k}>0\right\}}\left\{\pi_{j k}^{*}+r_{j k}\right\} .
$$

Observe that when computing the minimum among all sellers, we have to exclude those that do not have good $j$; otherwise, the price of good $j$ would be equal to 0 since, by assumption (1), if $q_{j k}=0$ then $r_{j k}=0$; and by (8), $\pi_{j k}^{*}=0$. Moreover, we define $p_{j}^{\left(\gamma^{*}, \pi^{*}\right)}$ to be the minimum because, even if $q_{j k}>0$, we may have that $A_{i j k}=0$ for all $i \in B$ in all optimal assignments $A \in F^{*}$; for instance, if $r_{j k}>v_{i j}$ for all $i \in B$.

Theorem 1 For every market $M, P^{*} \neq \varnothing$.

Proof Let $A^{*} \in F^{*}$ and $\left(\gamma^{*}, \pi^{*}\right) \in D^{*}$ be solutions of the (PLP) and (DLP), respectively. By Propositions 1 and 2 , they exist. To show that $P^{*} \neq \emptyset$, we will show that $\left(p^{\left(\gamma^{*}, \pi^{*}\right)}, A^{*}\right)$ is an equilibrium of $M$. We first show that for all $i \in B, A^{*}(i) \in D_{i}\left(p^{\left(\gamma^{*}, \pi^{*}\right)}\right)$.

Fix $i \in B$. Since $A^{*}$ is feasible, (D.a) and (D.b) hold.

Before proceeding, observe that by restriction (D.1) in the (DLP), for all $\left(j^{\prime}, k^{\prime}\right) \in G \times S$, $\gamma_{i}^{*}+\pi_{j^{\prime} k^{\prime}}^{*} \geq \tau_{i j^{\prime} k^{\prime}}$. Thus, $\gamma_{i}^{*} \geq \tau_{i j^{\prime} k^{\prime}}-\pi_{j^{\prime} k^{\prime}}^{*}$. If $\left(j^{\prime}, k^{\prime}\right) \in G \times S$ is such that $q_{j^{\prime} k^{\prime}}>0$ then, by (3), $\gamma_{i}^{*} \geq v_{i j^{\prime}}-\left(\pi_{j^{\prime} k^{\prime}}^{*}+r_{j^{\prime} k^{\prime}}\right)$. Thus, for all $j^{\prime} \in G$,

$$
\gamma_{i}^{*} \geq v_{i j^{\prime}}-\min _{\left\{k \in S \mid q_{j^{\prime} k}>0\right\}}\left\{\pi_{j^{\prime} k}^{*}+r_{j^{\prime} k}\right\} .
$$

To show that (D.c) holds assume that $\sum_{j k} A_{i j k}^{*}<d_{i}$. By (CS.2) of the Complementary Slackness Theorem,

$$
\gamma_{i}^{*}=0
$$

By (16) and (17), $\gamma_{i}^{*} \geq v_{i j}-p_{j}^{\left(\gamma^{*}, \pi^{*}\right)}$ for all $j \in G$. By (18), $0 \geq v_{i j}-p_{j}^{\left(\gamma^{*}, \pi^{*}\right)}$ for all $j \in G$. Hence, $\nabla_{i}^{>}\left(p^{\left(\gamma^{*}, \pi^{*}\right)}\right)=\emptyset$.

To show that (D.d) holds, fix $j \in G$ and assume that $\sum_{k} A_{i j k}^{*}>0$. We want to show that $j \in \nabla_{i}^{\geq}\left(p^{\left(\gamma^{*}, \pi^{*}\right)}\right)$. By assumption, there exists $k^{\prime} \in S$ such that $A_{i j k^{\prime}}^{*}>0$. Thus, $q_{j k^{\prime}}>0$. By (CS.1) of the Complementary Slackness Theorem, $\gamma_{i}^{*}+\pi_{j k^{\prime}}^{*}=\tau_{i j k^{\prime}}=v_{i j}-r_{j k^{\prime}}$. Thus, $\gamma_{i}^{*}=v_{i j}-\left(\pi_{j k^{\prime}}^{*}+r_{j k^{\prime}}\right)$. Hence, $\gamma_{i}^{*} \leq v_{i j}-\min _{\left\{k \in S \mid q_{j k}>0\right\}}\left\{\pi_{j k}^{*}+r_{j k}\right\}$. By (17), $\gamma_{i}^{*}=v_{i j}-\min _{\left\{k \in S \mid q_{j k}>0\right\}}\left\{\pi_{j k}^{*}+r_{j k}\right\}$. By (16),

$$
\gamma_{i}^{*}=v_{i j}-p_{j}^{\left(\gamma^{*}, \pi^{*}\right)} .
$$


By (16) and (17), $\gamma_{i}^{*} \geq v_{i j^{\prime}}-p_{j^{\prime}}^{\left(\gamma^{*}, \pi^{*}\right)}$ for all $j^{\prime} \in G$. By (19), $v_{i j}-p_{j}^{\left(\gamma^{*}, \pi^{*}\right)} \geq v_{i j^{\prime}}-p_{j^{\prime}}^{\left(\gamma^{*}, \pi^{*}\right)}$ for all $j^{\prime} \in G$. By restriction (D.2) in the (DLP), $\gamma_{i}^{*} \geq 0$. Hence, $j \in \nabla_{i}^{\geq}\left(p^{\left(\gamma^{*}, \pi^{*}\right)}\right)$.

To show that (E.S) holds fix $(j, k) \in G \times S$. We want to show that $\sum_{i} A_{i j k}^{*} \in S_{j k}\left(p_{j}^{\left(\gamma^{*}, \pi^{*}\right)}\right)$. Assume first that $\sum_{i} A_{i j k}^{*}<q_{j k}$. Then, by (CS.2) in the Complementary Slackness Theorem, $\pi_{j k}^{*}=0$. Since, by definition, $p_{j}^{\left(\gamma^{*}, \pi^{*}\right)}=\min _{\left\{k^{\prime} \in S \mid q_{j k^{\prime}}>0\right\}}\left\{\pi_{j k^{\prime}}^{*}+r_{j k^{\prime}}\right\}, p_{j} \leq \pi_{j k^{\prime}}^{*}+r_{j k^{\prime}}$ for all $k^{\prime}$ such that $q_{j k^{\prime}}>0$. But since $0 \leq \sum_{i} A_{i j k}^{*}<q_{j k}$ and $\left.\pi_{j k}^{*}=0, p_{j}^{\left(\gamma^{*}, \pi^{*}\right)}\right) \leq r_{j k}$. Hence, if $p_{j}>r_{j k}$ then $\sum_{i} A_{i j k}^{*} \in\left\{q_{j k}\right\}=S_{j k}\left(p_{j}^{\left(\gamma^{*}, \pi^{*}\right)}\right)$. If $p_{j}^{\left(\gamma^{*}, \pi^{*}\right)}=r_{j k}$ then (E.S) holds trivially since $\sum_{i} A_{i j k}^{*} \in\left\{0, \ldots, q_{j k}\right\}$. Assume $p_{j}^{\left(\gamma^{*}, \pi^{*}\right)}<r_{j k}$. By (1), $q_{j k}>0$. To get a contradiction, assume there exists $i \in B$ such that $A_{i j k}^{*}>0$. By (CS.1) of the Complementary Slackness Theorem, and since, by (3), $\tau_{i j k}=v_{i j}-r_{j k}, \gamma_{i}^{*}+\pi_{j k}^{*}=\tau_{i j k}=v_{i j}-r_{j k}$. By hypothesis, and since by restriction (D.3) of the (DLP), $\pi_{j k}^{*} \geq 0, \gamma_{i}^{*} \leq \gamma_{i}^{*}+\pi_{j k}^{*}<v_{i j}-p_{j}^{\left(\gamma^{*}, \pi^{*}\right)}$. Thus, $\gamma_{i}^{*}<v_{i j}-p_{j}^{\left(\gamma^{*}, \pi^{*}\right)}$, contradicting condition (17). Thus, for all $i \in B, A_{i j k}^{*}=0$. Hence, $\sum_{i} A_{i j k}^{*}=0 \in\{0\}=S_{j k}\left(p_{j}^{\left(\gamma^{*}, \pi^{*}\right)}\right)$. Thus $p^{\left(\gamma^{*}, \pi^{*}\right)} \in P^{*}$.

The proof of Theorem 1 (which proves that $P^{*}$ is non-empty by showing that for all $\left.\left(\gamma^{*}, \pi^{*}\right) \in D^{*}, p^{\left(\gamma^{*}, \pi^{*}\right)} \in P^{*}\right)$ implies the following corollary.

Corollary 2 Let $(\gamma, \pi) \in D^{*}$. Then, $p^{(\gamma, \pi)} \in P^{*}$.

Theorem 2 Let $M$ be a market and let $p \in \mathbb{R}_{+}^{n}$ be a price vector. The following two statements hold.

(2.1) Assume $p \in P^{*}$. Then, $A \in F^{*}$ if and only if $p$ and $A$ are compatible.

(2.2) $\quad p \in P^{*}$ if and only if $(\gamma(p), \pi(p)) \in D^{*}$.

Proof The statements of Theorem 2 will follow from Lemmata 2, 3, 4, and 5 below. We start by stating and proving a lemma that will be used in the proofs of Lemmata 4 and 5 .

Lemma 1 Assume $(\gamma(p), \pi(p)) \in D^{*}$ and $A \in F^{*}$. Then, $p$ and $A$ are compatible.

Proof of Lemma 1 Assume $p \in \mathbb{R}_{+}^{n}$ is such that $(\gamma(p), \pi(p)) \in D^{*}$ and $A \in F^{*}$. To show that $p$ and $A$ are compatible, we first show that for all $i \in B, A(i) \in D_{i}(p)$. Since $A$ is feasible, (D.a) and (D.b) hold. To show that (D.c) holds, assume $\nabla_{i}^{>}(p) \neq \emptyset$. Then, $v_{i j}-p_{j}>0$ for some $j \in G$. By definition, $\gamma_{i}(p)>0$. By condition (CS.2) in the Complementary Slackness Theorem, $\sum_{j k} A_{i j k}=d_{i}$; namely, condition (D.c) in the definition of $D_{i}(p)$ holds.

To show that (D.d) holds, fix $(i, j) \in B \times G$ and assume $\sum_{k} A_{i j k}>0$. We want to show that $j \in \nabla_{i}^{\geq}(p)$. Since $\sum_{k} A_{i j k}>0$, there exists a seller $k \in S$ such that $A_{i j k}>0$. Thus, $q_{j k}>0$ holds. Moreover, by condition (CS.1) in the Complementary Slackness Theorem, 
$\gamma_{i}(p)+\pi_{j k}(p)=\tau_{i j k}$. Since, by $(3), q_{j k}>0$ implies $\tau_{i j k}=v_{i j}-r_{j k}$, we have

$$
\gamma_{i}(p)+\pi_{j k}(p)+r_{j k}=v_{i j}
$$

We distinguish between the following two cases.

Case 1: $p_{j}-r_{j k} \geq 0$. Then, $\pi_{j k}(p)=p_{j}-r_{j k} \geq 0$. By $(20), \gamma_{i}(p)=v_{i j}-p_{j}$. If $\gamma_{i}(p)=$ $v_{i j}-p_{j}>0$ then $j \in \nabla_{i}^{>}(p)$. By $(7), j \in \nabla_{i}^{>}(p)$. If $\gamma_{i}(p)=v_{i j}-p_{j}=0$ then $\nabla_{i}^{>}(p)=\emptyset$. Hence, for all $\left(j^{\prime}, k^{\prime}\right) \in G \times S, 0 \geq v_{i j^{\prime}}-p_{j^{\prime}}$. Thus, $j \in \nabla_{i}^{\geq}(p)$.

Case 2: $p_{j}-r_{j k}<0$. Then, $\pi_{j k}(p)=0$. By $(20), \gamma_{i}(p)+r_{j k}=v_{i j}$. Hence, $\gamma_{i}(p)+p_{j}<v_{i j}$. Thus, $\gamma_{i}(p)<v_{i j}-p_{j}$. Hence, by definition of $\gamma_{i}(p)$, there exists $j^{\prime} \in \nabla_{i}^{>}(p)$ such that $\gamma_{i}(p)=v_{i j^{\prime}}-p_{j^{\prime}}<v_{i j}-p_{j}$, but this is impossible (i.e., Case 2 never occurs).

Hence, condition (D.d) holds for $i \in B$. Thus, $A(i) \in D_{i}(p)$ for all $i \in B$.

We want to show now that, for all $(j, k) \in G \times S, \sum_{i} A_{i j k} \in S_{j k}\left(p_{j}\right)$ holds. Fix $(j, k) \in$ $G \times S$. Since $A$ is feasible, $0 \leq \sum_{i} A_{i j k} \leq q_{j k}$. Assume $p_{j}=r_{j k}$. Then, $\sum_{i} A_{i j k} \in S_{j k}\left(p_{j}\right)$ holds trivially. Assume $p_{j}>r_{j k}$. Then, $\pi_{j k}(p)=p_{j}-r_{j k}>0$. By condition (CS.3) in the Complementary Slackness Theorem, $\sum_{i} A_{i j k}=q_{j k}$. Thus, $\sum_{i} A_{i j k} \in S_{j k}\left(p_{j}\right)=\left\{q_{j k}\right\}$. Finally, assume $p_{j}<r_{j k}$. Then, $\pi_{j k}(p)=0$ and $S_{j k}\left(p_{j}\right)=\{0\}$. Suppose $A_{i j k}>0$. Then, $q_{j k}>0$. By condition (CS.1) in the Complementary Slackness Theorem, $\gamma_{i}(p)+\pi_{j k}(p)=$ $\tau_{i j k}=v_{i j}-r_{j k} \geq 0$. Since $p_{j}<r_{j k}$,

$$
v_{i j}-p_{j}>v_{i j}-r_{j k}=\gamma_{i}(p) \geq 0,
$$

a contradiction with the definition of $\gamma_{i}(p)$. Thus, for all $i \in B, A_{i j k}=0$ and $\sum_{i} A_{i j k}=$ $0 \in S_{j k}\left(p_{j k}\right)=\{0\}$.

Lemma $2 \quad[\Longleftarrow$ of $(2.1)]$ Assume $p \in P^{*}$ and $A \in F$ are compatible. Then, $A \in F^{*}$.

Proof of Lemma 2 Let $p \in P^{*}$ and $A \in F$ be compatible. We show that $A \in F^{*}$ in two steps. We first show in Claim 1 that $(\gamma(p), \pi(p)) \in D$. Then, we show in Claim 2 that $T(A)=T^{d}(\gamma(p), \pi(p))$, and hence, by the Strong Duality Theorem, $A$ is a solution of the (PLP).

Claim 1: $(\gamma(p), \pi(p)) \in D$.

Proof of Claim 1 By their definitions, $\gamma_{i}(p) \geq 0$ for all $i \in B$ and $\pi_{j k}(p) \geq 0$ for all $(j, k) \in G \times S$; namely, restrictions (D.2) and (D.3) of the (DLP) hold. To show that, for all $(i, j, k) \in B \times G \times S$,

$$
\gamma_{i}(p)+\pi_{j k}(p) \geq \tau_{i j k}
$$


holds, fix $i \in B$ and assume first that $\gamma_{i}(p)=0$. Then, $v_{i j}-p_{j} \leq 0$ for all $j \in G$. If $q_{j k}>0$ then, by (3), $\tau_{i j k}=v_{i j}-r_{j k} \leq p_{j}-r_{j k} \leq \pi_{j k}(p)$. Thus, since $\gamma_{i}(p)=0$, (21) holds. If $q_{j k}=0$ then, by (3), $\tau_{i j k}=0$. Thus, by definition of $\pi_{j k}(p)$ and since $\gamma_{i}(p)=0,(21)$ holds. Hence, if $\gamma_{i}(p)=0$ then (21) holds.

Assume now $\gamma_{i}(p)>0$. Then, there exists $j \in \nabla_{i}^{>}(p)$ such that $\gamma_{i}(p)=v_{i j}-p_{j}>0$. By definition of $\nabla_{i}^{>}(p)$, for all $\left(j^{\prime}, k^{\prime}\right) \in G \times S$,

$$
\begin{aligned}
v_{i j}-p_{j}+\pi_{j^{\prime} k^{\prime}}(p) & \geq v_{i j^{\prime}}-p_{j^{\prime}}+\pi_{j^{\prime} k^{\prime}}(p) \\
& \geq v_{i j^{\prime}}-p_{j^{\prime}}+p_{j^{\prime}}-r_{j^{\prime} k^{\prime}} \\
& =v_{i j^{\prime}}-r_{j^{\prime} k^{\prime}} .
\end{aligned}
$$

If $q_{j^{\prime} k^{\prime}}>0$ then, by (3), $\tau_{i j^{\prime} k^{\prime}}=v_{i j^{\prime}}-r_{j^{\prime} k^{\prime}}$ and hence, $v_{i j}-p_{j}+\pi_{j^{\prime} k^{\prime}}(p) \geq \tau_{i j^{\prime} k^{\prime}}$. If $q_{j^{\prime} k^{\prime}}=0$ then $\tau_{i j^{\prime} k^{\prime}}=0$, and since $v_{i j}-p_{j}>0$ and $\pi_{j^{\prime} k^{\prime}}(p) \geq 0, v_{i j}-p_{j}+\pi_{j^{\prime} k^{\prime}}(p) \geq \tau_{i j^{\prime} k^{\prime}}$ holds as well. Thus, for all $\left(i, j^{\prime}, k^{\prime}\right) \in B \times G \times S, \gamma_{i}(p)+\pi_{j^{\prime} k^{\prime}}(p) \geq \tau_{i j^{\prime} k^{\prime}}$. Hence, (21) holds as well when $\gamma_{i}(p)>0$. Thus, $(\gamma(p), \pi(p)) \in D$. This ends the proof of Claim 1.

Claim 2: $T(A)=T^{d}(\gamma(p), \pi(p))$.

Proof of Claim 2: By definition,

$$
T(A)=\sum_{i j k} \tau_{i j k} \cdot A_{i j k}
$$

Condition (E.D) in the definition of an equilibrium price vector implies that, for every $i \in B, A(i) \in D_{i}(p)$. Fix $(i, j, k) \in B \times G \times S$ and assume $A_{i j k}>0$. By condition (D.d) in the definition of $D_{i}(p), j \in \nabla_{i}^{\geq}(p)$. Observe that $A_{i j k}>0$ implies $q_{j k}>0$. Thus, by (3),

$$
\tau_{i j k}=v_{i j}-r_{j k}
$$

If $\nabla_{i}^{>}(p) \neq \emptyset$ then $j \in \nabla_{i}^{\geq}(p)$ implies $v_{i j}-p_{j} \geq v_{i j^{\prime}}-p_{j^{\prime}}$ for all $j^{\prime} \in G$. Thus, $j \in \nabla_{i}^{>}(p)$. Hence, by definitions of $\gamma_{i}(p)$ and $\nabla_{i}^{>}(p)$, and condition (23),

$$
\begin{aligned}
\gamma_{i}(p)+p_{j}-r_{j k} & =v_{i j}-p_{j}+p_{j}-r_{j k} \\
& =v_{i j}-r_{j k} \\
& =\tau_{i j k} .
\end{aligned}
$$

If $\nabla_{i}^{>}(p)=\emptyset$ then, since $j \in \nabla_{i}^{\geq}(p), v_{i j}-p_{j}=0$ and $\gamma_{i}(p)=0$. Hence, by $(23)$,

$$
\begin{aligned}
\gamma_{i}(p)+p_{j}-r_{j k} & =p_{j}-r_{j k} \\
& =v_{i j}-r_{j k} \\
& =\tau_{i j k} .
\end{aligned}
$$


Thus, $\gamma_{i}(p)+p_{j}-r_{j k}=\tau_{i j k}$. Hence, for all $(i, j, k) \in B \times G \times S$ such that $A_{i j k}>0$, $\tau_{i j k} \cdot A_{i j k}=\left(\gamma_{i}(p)+p_{j}-r_{j k}\right) \cdot A_{i j k}$. From (22),

$$
\begin{aligned}
T(A) & =\sum_{i j k} \tau_{i j k} \cdot A_{i j k} \\
& =\sum_{i j k}\left(\gamma_{i}(p)+p_{j}-r_{j k}\right) \cdot A_{i j k} \\
& =\sum_{i j k} \gamma_{i}(p) \cdot A_{i j k}+\sum_{i j k}\left(p_{j}-r_{j k}\right) \cdot A_{i j k} .
\end{aligned}
$$

Thus,

$$
T(A)=\sum_{i}\left(\sum_{j k} A_{i j k}\right) \cdot \gamma_{i}(p)+\sum_{j k}\left(\sum_{i} A_{i j k}\right) \cdot\left(p_{j}-r_{j k}\right) .
$$

Fix $i \in B$. By condition (D.c) in the definition of $D_{i}(p)$, if $\sum_{j k} A_{i j k}<d_{i}$ then $\nabla_{i}^{>}(p)=\emptyset$, and by the definition of $\gamma_{i}(p), \gamma_{i}(p)=0$. Hence, by (24),

$$
T(A)=\sum_{i} d_{i} \cdot \gamma_{i}(p)+\sum_{j k}\left(\sum_{i} A_{i j k}\right) \cdot\left(p_{j}-r_{j k}\right) .
$$

Condition (E.S) in the definition of an equilibrium price vector implies that, for every $(j, k) \in G \times S, \sum_{i} A_{i j k} \in S_{j k}\left(p_{j}\right)$. To show that, for all $(j, k) \in G \times S$,

$$
\left(\sum_{i} A_{i j k}\right) \cdot\left(p_{j}-r_{j k}\right)=q_{j k} \cdot \pi_{j k}(p)
$$

holds, we distinguish among several cases.

Case 1: $q_{j k}=0$. Then, by supply feasibility, $\sum_{i} A_{i j k}=0$. Thus, (26) holds.

Case 2: $q_{j k}>0$.

Case 2.1: $\sum_{i} A_{i j k}=q_{j k}$. Then, by (E.S), $p_{j}-r_{j k} \geq 0$. Hence, $p_{j}-r_{j k}=\pi_{j k}(p)$. Thus, (26) holds.

Case 2.2: $0<\sum_{i} A_{i j k}<q_{j k}$. Then, by (E.S), $p_{j}=r_{j k}$. Hence, $\pi_{j k}(p)=p_{j}-r_{j k}=0$. Thus, (26) holds.

Case 2.3: $\sum_{i} A_{i j k}=0$. Then, by (E.S), $p_{j} \leq r_{j k}$. Hence, $\pi_{j k}(p)=0$. Thus, (26) holds. Hence, for all $(j, k) \in G \times S,(26)$ holds. Thus, by $(25), T(A)=\sum_{i} d_{i} \cdot \gamma_{i}(p)+\sum_{j k} q_{j k} \cdot \pi_{j k}(p)$. Therefore, $T(A)=T^{d}(\gamma(p), \pi(p))$.

The statement of Lemma 2 follows from Claims 1 and 2.

Lemma $3 \quad[\Longrightarrow$ of $(2.2)]$ Assume $p \in P^{*}$. Then, $(\gamma(p), \pi(p)) \in D^{*}$.

Proof of Lemma 3 Assume $p \in P^{*}$ and let $A \in F$ be any assignment compatible with $p$. Thus, the hypothesis of Lemma 2 hold. By Claims 1 and 2 in the proof of Lemma $2,(\gamma(p), \pi(p)) \in D$ and $T(A)=T^{d}(\gamma(p), \pi(p))$. By the Strong Duality Theorem, $(\gamma(p), \pi(p)) \in D^{*}$. 
Lemma $4[\Longrightarrow$ of $(2.1)]$ Assume $p \in P^{*}$ and $A \in F^{*}$. Then, $p$ and $A$ are compatible.

Proof of Lemma 4 Follows from Lemmata 1 and 3.

Lemma $5 \quad[\Longleftarrow$ of $(2.2)]$ Assume $(\gamma(p), \pi(p)) \in D^{*}$. Then, $p \in P^{*}$.

Proof of Lemma 5 Let $p \in \mathbb{R}_{+}^{n}$ be such that $(\gamma(p), \pi(p)) \in D^{*}$. We want to show that $p$ is an equilibrium price vector of $M$. Let $A \in F^{*}$ be arbitrary. By Lemma $1, p$ and $A$ are compatible. Hence, by definition, $p \in P^{*}$.

Theorem 2 holds since condition (2.1) follows from Lemmata 2 and 4, and condition (2.2) follows from Lemmata 3 and 5.

Proposition 5 Let $M$ be a market and let $p \in P^{*}$. Then, $\bar{p} \in P^{*}$.

Proof Let $A \in F^{*}$ be an optimal assignment of $M$. We will prove that $(\bar{p}, A)$ is a competitive equilibrium of $M$ by showing that conditions (E.D) and (E.S) of Definition 3 are satisfied by $\bar{p}$ with respect to $A$.

(E.D) For every $i \in B, A(i) \in D_{i}(\bar{p})$.

Fix $i \in B$. Since $A$ is feasible, conditions (D.a) and (D.b) hold.

To show that condition (D.c) holds, assume $\nabla_{i}^{>}(\bar{p}) \neq \emptyset$. Then, there exists $j \in \nabla_{i}^{>}(\bar{p})$ such that $v_{i j}-\bar{p}_{j}>0$. Since either $\bar{p}_{j}=p_{j}$ or $\bar{p}_{j}=p_{j}^{S}$ we have that either $0<v_{i j}-\bar{p}_{j}=$ $v_{i j}-p_{j}$ or $0<v_{i j}-\bar{p}_{j}=v_{i j}-p_{j}^{S}$, which implies that either $\nabla_{i}^{>}(p) \neq \emptyset$ or $\nabla_{i}^{>}\left(p^{S}\right) \neq \emptyset$. Since $p$ and $p^{S}$ are both compatible with $A, \sum_{j k} A_{i j k}=d_{i}$. Thus condition (D.c) holds for $\bar{p}$.

To show that condition (D.d) holds, let $(i, j) \in B \times G$ be such that $\sum_{k} A_{i j k}>0$. Thus, $j \in G^{>}$. We have to show that $j \in \nabla_{i}^{\geq}(\bar{p})$. Since $p$ and $p^{S}$ are both compatible with $A$, $j \in \nabla_{i}^{\geq}(p) \cap \nabla_{i}^{\geq}\left(p^{S}\right)$. By definition of $\nabla_{i}^{\geq}(p)$,

$$
v_{i j}-p_{j} \geq 0
$$

and

$$
v_{i j}-p_{j} \geq v_{i j^{\prime}}-p_{j^{\prime}} \text { for every } j^{\prime} \in G \text {. }
$$

By definition of $\nabla_{i}^{\geq}\left(p^{S}\right), v_{i j}-p_{j}^{S} \geq 0$ and $v_{i j}-p_{j}^{S} \geq v_{i j^{\prime}}-p_{j^{\prime}}^{S}=v_{i j^{\prime}}-\max _{p \in P^{*}} p_{j^{\prime}}$ for every $j^{\prime} \in G$. We next show that:

$$
v_{i j}-\bar{p}_{j} \geq 0
$$

and

$$
v_{i j}-\bar{p}_{j} \geq v_{i j^{\prime}}-\bar{p}_{j^{\prime}} \text { for every } j^{\prime} \in G \text {. }
$$


Since $j \in G^{>}, \bar{p}_{j}=p_{j}$. Thus, by (27), $v_{i j}-\bar{p}_{j} \geq 0$. We distinguish between the following two cases.

Case 1: $j^{\prime} \in G^{>}$. Then, $\bar{p}_{j^{\prime}}=p_{j^{\prime}}$ and

$$
\begin{aligned}
v_{i j}-\bar{p}_{j} & =v_{i j}-p_{j} \quad \text { by definition of } \bar{p}_{j} \\
& \geq v_{i j^{\prime}}-p_{j^{\prime}} \quad \text { by }(28) \\
& =v_{i j^{\prime}}-\bar{p}_{j^{\prime}} \quad \text { by definition of } \bar{p}_{j^{\prime}} .
\end{aligned}
$$

Hence, $v_{i j}-\bar{p}_{j} \geq v_{i j^{\prime}}-\bar{p}_{j^{\prime}}$ for every $j^{\prime} \in G^{>}$.

Case $2: j^{\prime} \notin G^{>}$. Then, $\bar{p}_{j^{\prime}}=p_{j^{\prime}}^{S}=\max _{p \in P^{*}} p_{j^{\prime}}$ and

$$
\begin{aligned}
v_{i j}-\bar{p}_{j} & =v_{i j}-p_{j} & & \text { by definition of } \bar{p}_{j} \\
& \geq v_{i j^{\prime}}-p_{j^{\prime}} & & \text { by }(28) \\
& \geq v_{i j^{\prime}}-\max _{p \in P^{*}} p_{j^{\prime}} & & \\
& =v_{i j^{\prime}}-\bar{p}_{j^{\prime}} & & \text { by definition of } \bar{p}_{j^{\prime}} .
\end{aligned}
$$

Hence, $v_{i j}-\bar{p}_{j} \geq v_{i j^{\prime}}-\bar{p}_{j^{\prime}}$ for every $j^{\prime} \notin G^{>}$.

Thus, $j \in \nabla_{i}^{\geq}(\bar{p})$.

(E.S) For every $j \in G, \sum_{i} A_{i j k} \in S_{j k}\left(\bar{p}_{j}\right)$.

Assume first that $j \in G^{>}$. Then, $\bar{p}_{j}=p_{j}$ and $S_{j k}\left(\bar{p}_{j}\right)=S_{j k}\left(p_{j}\right)$. Since $p$ and $A$ are compatible, $\sum_{i} A_{i j k} \in S_{j k}\left(p_{j}\right)$. Thus, $\sum_{i} A_{i j k} \in S_{j k}\left(\bar{p}_{j}\right)$. Assume now that $j \notin G^{>}$. Then, $\bar{p}_{j}=p_{j}^{S}$ and $S_{j k}\left(\bar{p}_{j}\right)=S_{j k}\left(p_{j}^{S}\right)$. Since $p^{S}$ and $A$ are compatible, $\sum_{i} A_{i j k} \in S_{j k}\left(p_{j}^{S}\right)$. Thus, $\sum_{i} A_{i j k} \in S_{j k}\left(\bar{p}_{j}\right)$.

Proposition 6 For every $p \in P^{*}, p^{(\gamma(p), \pi(p))}=\bar{p}$.

Proof Let $p \in P^{*}$ be given and let $A^{*} \in F^{*}$ be any compatible assignment. By definition, for all $j \in G, \tilde{p}_{j} \equiv p_{j}^{(\gamma(p), \pi(p))}=\min _{\left\{k \in K \mid q_{j k}>0\right\}}\left\{\pi_{j k}(p)+r_{j k}\right\}$.

Assume first that $j \notin G^{>}$. Then, $\sum_{i k} A_{i j k}^{*}=0$. By (CS.2) of the Complementary Slackness Theorem, $\pi_{j k}^{*}=0$ for all $k \in S$ and all $\pi_{j k}^{*}$ such that there exists $\gamma^{*}$ with the property that $\left(\gamma^{*}, \pi^{*}\right) \in D^{*}$. Thus, by part $(2.2)$ of Theorem $2, \pi_{j k}(p)=0$. Hence, $\tilde{p}_{j}=$ $\min _{\left\{k \in K \mid q_{j k}>0\right\}} r_{j k}$. By Corollary 2 and definition of $p^{S}, \tilde{p}_{j} \leq p_{j}^{S}$. To obtain a contradiction, assume $\tilde{p}_{j}<p_{j}^{S}$. Then, there exists $k \in K$ such that $q_{j k}>0$ and $r_{j k}<p_{j}^{S}$. Since, by Milgrom (2009), $P^{*}$ is a complete lattice, $p^{S} \in P^{*}$, (E.S) implies that $\sum_{i} A_{i j k}^{*}=q_{j k}>0$, a contradiction.

Assume now that $j \in G^{>}$. It is immediate to see that, for all $p^{\prime} \in P^{*}$,

$$
G^{>} \subseteq \bigcup_{i \in B} \nabla_{i}^{\geq}\left(p^{\prime}\right)
$$


holds. Next, we show that the following claim holds.

Claim Let $p^{\prime} \in P^{*}$ and $(i, j) \in B \times G$ be such that $j \in \nabla_{i}^{\geq}\left(p^{\prime}\right)$, then $v_{i j}-p_{j}^{\prime}=\gamma_{i}\left(p^{\prime}\right)$.

Proof of Claim Since $j \in \nabla_{i}^{\geq}\left(p^{\prime}\right), v_{i j}-p_{j}^{\prime} \geq 0$ and for all $j^{\prime} \in G, v_{i j}-p_{j}^{\prime} \geq v_{i j^{\prime}}-p_{j^{\prime}}^{\prime}$. If $v_{i j}-p_{j}^{\prime}=0$, then $v_{i j^{\prime}}-p_{j^{\prime}}^{\prime} \leq 0$ for all $j^{\prime} \in G$. Thus, $\gamma_{i}\left(p^{\prime}\right)=0=v_{i j}-p_{j}^{\prime}$. If $v_{i j}-p_{j}^{\prime}>0$, then $j \in \nabla_{i}^{>}\left(p^{\prime}\right)$. Thus, $\gamma_{i}\left(p^{\prime}\right)=v_{i j}-p_{j}^{\prime}$.

By restriction (D.1) of the (DLP), for all $(\gamma, \pi) \in D^{*}$ and all $(i, j, k) \in B \times G \times S$, $\gamma_{i}+\pi_{j k} \geq \tau_{i j k}$. Thus, by (3), for all $i \in B$ and all $(j, k)$ such that $q_{j k}>0, \gamma_{i}+\pi_{j k} \geq v_{i j}-r_{j k}$. Hence,

$$
\pi_{j k}+r_{j k} \geq v_{i j}-\gamma_{i}
$$

Since $j \in G^{>}$, condition (29) implies that there exist $\left(i^{\prime}, k^{\prime}\right) \in B \times S$ such that $q_{j k^{\prime}}>0$, $A_{i^{\prime} j k^{\prime}}^{*}>0$ and $j \in \nabla_{i}^{\geq}\left(p^{\prime}\right)$. Thus, by (30) applied to $\left(\gamma\left(p^{\prime}\right), \pi\left(p^{\prime}\right)\right)$ and $i^{\prime} \in B, \pi_{j k}\left(p^{\prime}\right)+r_{j k} \geq$ $v_{i^{\prime} j}-\gamma_{i^{\prime}}\left(p^{\prime}\right)$ for all $k \in S$ such that $q_{j k}>0$. By the claim above, $\pi_{j k}\left(p^{\prime}\right)+r_{j k} \geq v_{i^{\prime} j}-\gamma_{i^{\prime}}\left(p^{\prime}\right)=$ $p_{j}^{\prime}$. Thus,

$$
\min _{\left\{k \in K \mid q_{j k}>0\right\}}\left\{\pi_{j k}\left(p^{\prime}\right)+r_{j k}\right\} \geq p_{j}^{\prime} .
$$

Moreover, by (CS.1) of the Complementary Slackness Theorem, $\gamma_{i^{\prime}}\left(p^{\prime}\right)+\pi_{j k^{\prime}}\left(p^{\prime}\right)=\tau_{i^{\prime} j k^{\prime}}=$ $v_{i^{\prime} j}-r_{j k^{\prime}}$. Thus, $\pi_{j k^{\prime}}\left(p^{\prime}\right)+r_{j k^{\prime}}=v_{i^{\prime} j}-\gamma_{i^{\prime}}\left(p^{\prime}\right)$, and by the claim above, $\pi_{j k^{\prime}}\left(p^{\prime}\right)+r_{j k^{\prime}}=$ $v_{i^{\prime} j}-\gamma_{i^{\prime}}\left(p^{\prime}\right)=p_{j}^{\prime}$. Thus, by $(31), \min _{\left\{k \in K \mid q_{j k}>0\right\}}\left\{\pi_{j k}\left(p^{\prime}\right)+r_{j k}\right\}=p_{j}^{\prime}$, which implies that $p_{j}^{\prime}=\bar{p}_{j}$. Hence, $p^{(\gamma(p), \pi(p))}=\bar{p}$.

Theorem 3 The four-tuple $\left(P^{* *}, \geq, \vee, \wedge\right)$ is a complete lattice.

Proof We will show that the set of restricted equilibrium price vectors $P^{* *}$ of $M$ has the property that each of its non-empty subsets has a supremum and an infimum according to the order $\geq$. This will imply that the set $P^{* *}$ has a complete lattice structure. Let $Z \subseteq P^{* *}$ be a non-empty subset of restricted equilibrium price vectors of $M$. Define the price vector $p^{B}(Z) \in \mathbb{R}_{+}^{n}$ by taking, for each $j \in G$, the infimum among all $j$-components of the vectors in $Z$. Similarly, define the price vector $p^{S}(Z) \in \mathbb{R}_{+}^{n}$ by taking, for each $j \in G$, the supremum among all $j$-components of the vectors in $Z$. Then, it turns out that $p^{B}(Z)$ and $p^{S}(Z)$ are also restricted equilibrium price vectors of $M$ (see Lemma 7 below). Formally, given a market $M$, define $p^{B}(Z)=\left(p_{j}^{B}(Z)\right)_{j \in G} \in \mathbb{R}_{+}^{n}$ and $p^{S}(Z)=\left(p_{j}^{S}(Z)\right)_{j \in G} \in \mathbb{R}_{+}^{n}$ as follows: for each $j \in G$, let

$$
p_{j}^{B}(Z)=\inf _{p \in Z} p_{j} \quad \text { and } \quad p_{j}^{S}(Z)=\sup _{p \in Z} p_{j} .
$$

For the special case where $Z=P^{*}$, we have written $p^{B}$ and $p^{S}$ instead of $p^{B}\left(P^{*}\right)$ and $p^{S}\left(P^{*}\right)$. 
Lemma 7 Let $M$ be a market. Then, $p^{B}(Z), p^{S}(Z) \in P^{* *}$ for all $\emptyset \neq Z \subseteq P^{* *}$.

Proof of Lemma 7 Let $A \in F^{*}$ be an optimal assignment of $M$. Given a non empty subset $Z \subseteq P^{* *}$, we will first prove that $p^{B}(Z)$ and $p^{S}(Z)$ are equilibrium price vectors of $M$ by showing that conditions (E.D) and (E.S) of Definition 3 are satisfied by $p^{B}(Z)$ and $p^{S}(Z)$ with respect to $A$.

(E.D) for $p^{S}(Z)$ : For every $i \in B, A(i) \in D_{i}\left(p^{S}(Z)\right)$.

Fix $i \in B$. Since $A$ is feasible, conditions (D.a) and (D.b) hold.

To show that condition (D.c) holds, assume $\nabla_{i}^{>}\left(p^{S}(Z)\right) \neq \emptyset$. Then, there exists $j \in$ $\nabla_{i}^{>}\left(p^{S}(Z)\right)$ such that $v_{i j}-p_{j}^{S}(Z)>0$. Since $p_{j}^{S}(Z)=\sup _{p \in Z} p_{j}$, we have that for every $p \in Z, 0<v_{i j}-p_{j}^{S}(Z) \leq v_{i j}-p_{j}$, which implies that $\nabla_{i}^{>}(p) \neq \emptyset$. Because $p$ and $A$ are compatible, $\sum_{j k} A_{i j k}=d_{i}$. Thus condition (D.c) holds for $p^{S}(Z)$.

To show that condition (D.d) holds, let $j \in G$ be such that $\sum_{k} A_{i j k}>0$. We have to show that $j \in \nabla_{i}^{\geq}\left(p^{S}(Z)\right)$. Since for all $p \in Z, p$ and $A$ are compatible, $j \in \nabla_{i}^{\geq}(p)$ for every $p \in Z$. By definition of $\nabla_{i}^{\geq}(p), v_{i j}-p_{j} \geq 0$ and $v_{i j}-p_{j} \geq v_{i j^{\prime}}-p_{j^{\prime}}$ for every $j^{\prime} \in G$. For every $j^{\prime} \in G$,

$$
v_{i j^{\prime}}-p_{j^{\prime}} \geq v_{i j^{\prime}}-\sup _{\hat{p} \in Z} \hat{p}_{j^{\prime}}
$$

holds for all $p \in Z$. Let $\left\{p^{m}\right\}_{m \in \mathbb{N}}$ be a sequence such that, for all $m \in \mathbb{N}, p^{m} \in Z$ and $\left\{p_{j}^{m}\right\}_{m \in \mathbb{N}} \rightarrow \sup _{p \in Z} p_{j}$. By $(33), v_{i j^{\prime}}-p_{j^{\prime}}^{m} \geq v_{i j^{\prime}}-\sup _{p \in Z} p_{j^{\prime}}$ for all $m \in \mathbb{N}$. Since $j \in \nabla_{i}^{\geq}(p)$ for every $p \in Z, j \in \nabla_{i}^{\geq}\left(p^{m}\right)$ for every $m \in \mathbb{N}$. Thus, $v_{i j}-p_{j}^{m} \geq v_{i j^{\prime}}-p_{j^{\prime}}^{m}$ for all $m \in \mathbb{N}$. Thus, $v_{i j}-p_{j}^{m} \geq v_{i j^{\prime}}-\sup _{p \in Z} p_{j^{\prime}}$ for all $m \in \mathbb{N}$. Hence, $v_{i j}-\sup _{p \in Z} p_{j} \geq v_{i j^{\prime}}-\sup _{p \in Z} p_{j^{\prime}}$. Thus, $j \in \nabla_{i}^{\geq}\left(p^{S}(Z)\right)$.

(E.S) for $p^{S}(Z)$ : For every $(j, k) \in G \times S, \sum_{i} A_{i j k} \in S_{j k}\left(p_{j}^{S}(Z)\right)$.

Fix $(j, k) \in G \times S$. If $p_{j}^{S}(Z)<r_{j k}$ then, for all $p \in Z, p_{j} \leq p_{j}^{S}(Z)<r_{j k}$. Thus, $\sum_{i} A_{i j k}=0 \in S_{j k}\left(p_{j}\right)$. Thus, $\sum_{i} A_{i j k} \in\{0\}=S_{j k}\left(p_{j}^{S}(Z)\right)$. If $p_{j}^{S}(Z)>r_{j k}$, let $\left\{p^{m}\right\}_{m \in \mathbb{N}}$ be a sequence such that, for all $m \in \mathbb{N}, p^{m} \in Z$ and $\left\{p_{j}^{m}\right\}_{m \in \mathbb{N}} \rightarrow \sup _{p \in Z} p_{j}$. Then, there exists $\bar{m} \in \mathbb{N}$ such that for all $m>\bar{m}, p_{j}^{m}>r_{j k}$. Thus, $\sum_{i} A_{i j k} \in\left\{q_{j k}\right\}=S_{j k}\left(p_{j}^{m}\right)$ for all $m>\bar{m}$. Hence, $\sum_{i} A_{i j k} \in\left\{q_{j k}\right\}=S_{j k}\left(p_{j}^{S}(Z)\right)$.

(E.D) for $p^{B}(Z)$ : For every $i \in B, A(i) \in D_{i}\left(p^{B}(Z)\right)$.

Fix $i \in B$. Since $A$ is feasible, conditions (D.a) and (D.b) hold.

To show that condition (D.c) holds, assume $\nabla_{i}^{>}\left(p^{B}(Z)\right) \neq \emptyset$. Then, there exists $j \in$ $\nabla_{i}^{>}\left(p^{B}(Z)\right)$ such that $v_{i j}-p_{j}^{B}(Z)>0$. Let $\left\{p^{m}\right\}_{m \in \mathbb{N}}$ be a sequence such that, for all $m \in \mathbb{N}, p^{m} \in Z$ and $\left\{p_{j}^{m}\right\}_{m \in \mathbb{N}} \rightarrow \inf _{p \in Z} p_{j}$. Then, there exist $\bar{m} \in \mathbb{N}$ such that, for all 
$m>\bar{m}, v_{i j}-p_{j}^{m}>0$, which implies that $\nabla_{i}^{>}\left(p^{m}\right) \neq \emptyset$. Because $p^{m}$ and $A$ are compatible, $\sum_{j k} A_{i j k}=d_{i}$. Thus, condition (D.c) holds for $p^{B}(Z)$.

To show that condition (D.d) holds, let $j \in G$ be such that $\sum_{k} A_{i j k}>0$. We have to show that $j \in \nabla_{i}^{\geq}\left(p^{B}(Z)\right)$. Since for all $p \in Z, p$ and $A$ are compatible, $j \in \nabla_{i}^{\geq}(p)$ for every $p \in Z$. By definition of $\nabla_{i}^{\geq}(p), v_{i j}-p_{j} \geq 0$ and $v_{i j}-p_{j} \geq v_{i j^{\prime}}-p_{j^{\prime}}$ for every $j^{\prime} \in G$. By definition of $p^{B}(Z), v_{i j}-p_{j}^{B}(Z)=v_{i j}-\inf _{p \in Z} p_{j} \geq v_{i j}-p_{j}$ for all $p \in Z$. Fix $j^{\prime} \in G$ and let $\left\{p^{m}\right\}_{m \in \mathbb{N}}$ be a sequence such that, for all $m \in \mathbb{N}, p^{m} \in Z$ and $\left\{p_{j^{\prime}}^{m}\right\}_{m \in \mathbb{N}} \rightarrow \inf _{p \in Z} p_{j^{\prime}}$. Then, by definition of $p_{j}^{B}(Z), v_{i j}-p_{j}^{B}(Z) \geq v_{i j^{\prime}}-p_{j^{\prime}}^{m}$ for every $m \in \mathbb{N}$. Since $j \in \nabla_{i}^{\geq}\left(p^{m}\right)$, $v_{i j}-p_{j}^{m} \geq v_{i j^{\prime}}-p_{j^{\prime}}^{m}$ for every $m \in \mathbb{N}$. Thus, $v_{i j}-p_{j}^{B}(Z) \geq v_{i j^{\prime}}-p_{j^{\prime}}^{m}$ for every $m \in \mathbb{N}$. Hence, $v_{i j}-p_{j}^{B}(Z) \geq v_{i j^{\prime}}-p_{j^{\prime}}^{B}(Z)$. Since this holds for all $j^{\prime} \in G, j \in \nabla_{i}^{\geq}\left(p^{B}(Z)\right)$.

(E.S) for $p^{B}(Z)$ : For every $(j, k) \in G \times S, \sum_{i} A_{i j k} \in S_{j k}\left(p_{j}^{B}(Z)\right)$.

Fix $(j, k) \in G \times S$. If $p_{j}^{B}(Z)>r_{j k}$ then, $p_{j}>r_{j k}$ for all $p \in Z$. Thus, $\sum_{i} A_{i j k}=q_{j k} \in$ $\left\{q_{j k}\right\}=S_{j k}\left(p_{j}\right)$. Thus, $\sum_{i} A_{i j k} \in S_{j k}\left(p_{j}^{B}(Z)\right)$. If $p_{j}^{S}<r_{j k}$, let $\left\{p^{m}\right\}_{m \in \mathbb{N}}$ be a sequence such that, for all $m \in \mathbb{N}, p^{m} \in Z$ and $\left\{p_{j}^{m}\right\}_{m \in \mathbb{N}} \rightarrow \inf _{p \in Z} p_{j}$. Then, there exists $\bar{m} \in \mathbb{N}$ such that for all $m>\bar{m}, p_{j}^{m}<r_{j k}$. Thus, $\sum_{i} A_{i j k} \in\{0\}=S_{j k}\left(p_{j}^{m}\right)$ for all $m>\bar{m}$. Hence, $\sum_{i} A_{i j k} \in\{0\}=S_{j k}\left(p_{j}^{B}(Z)\right)$.

We now prove that indeed $p^{B}(Z), p^{S}(Z) \in P^{* *}$. That is, that $p_{j}^{B}(Z)=p_{j}^{S}$ and $p_{j}^{S}(Z)=p_{j}^{S}$ for every $j \notin G^{>}$. Let $j \notin G^{>}$. Since $p_{j}^{B}(Z)=\inf _{p \in Z} p_{j}$ and $p \in Z \subseteq P^{* *}$ implies $p_{j}=p_{j}^{S}$, $\inf _{p \in Z} p_{j}=p_{j}^{S}$. Thus, $p_{j}^{B}(Z)=p_{j}^{S}$. Hence, $p^{B}(Z) \in P^{* *}$. Similarly, $p^{S}(Z) \in P^{* *}$.

By Lemma 7 above we can write for each $\emptyset \neq Z \subseteq P^{* *}$ and $j \in G, p_{j}^{S}(Z)=\max _{p \in Z} p_{j}$ and $p_{j}^{B}(Z)=\min _{p \in Z} p_{j}$. In particular, $p_{j}^{S}\left(P^{* *}\right)=\max _{p \in P^{* *}} p_{j}$ for all $j \in G$ and $p_{j}^{B}\left(P^{* *}\right)=$ $\min _{p \in P^{* *}} p_{j}$ for all $j \in G^{>}$and $p_{j}^{B}=p_{j}^{S}$ for all $j \notin G^{>}$.

To show that $\left(P^{* *}, \geq, \vee, \wedge\right)$ is a lattice let $p, p^{\prime} \in P^{* *}$ and set $Z=\left\{p, p^{\prime}\right\}$. Then, $p \vee p^{\prime}=p^{S}(Z)$ and $p \wedge p^{\prime}=p^{B}(Z)$. By Lemma $7, p \vee p^{\prime} \in P^{* *}$ and $p \wedge p^{\prime} \in P^{* *}$. Moreover, it is immediate to check that $\vee$ and $\wedge$ are idempotent, commutative, associative, and absorbing binary operations on $P^{* *}$. Thus, $\left(P^{* *}, \geq, \vee, \wedge\right)$ is a lattice. To prove that it is complete, consider any $\emptyset \neq Z \subseteq P^{* *}$. By definition, $l u b_{\succeq} Z=p^{S}(Z)$ and $l l b_{\succeq} Z=p^{B}(Z)$. By Lemma $7, p^{S}(Z), p^{B}(Z) \in P^{* *}$. Thus, $\left(P^{* *}, \geq, \vee, \wedge\right)$ is a complete lattice.

Proposition 7 Let $p, p^{\prime} \in P^{* *}$ be two restricted equilibrium price vectors of market $M$. Then,

$$
u_{i}(p) \geq u_{i}\left(p^{\prime}\right) \text { for every } i \in B \text { if and only if } p_{j}^{\prime} \geq p_{j} \text { for every } j \in G .
$$

Proof It follows from definition of $P^{* *}$ and Lemma 8 below. 
Lemma 8 Let $p, p^{\prime} \in P^{*}$ be two equilibrium price vectors of market $M$. Then, $u_{i}(p) \geq u_{i}\left(p^{\prime}\right)$ for every $i \in B$ if and only if $p_{j}^{\prime} \geq p_{j}$ for every $j \in G^{>}$.

Proof of Lemma 8 Let $p, p^{\prime} \in P^{*}$.

$\Longrightarrow)$ Assume $u_{i}(p) \geq u_{i}\left(p^{\prime}\right)$ for every $i \in B$. By $(13), \gamma_{i}(p) \geq \gamma_{i}\left(p^{\prime}\right)$ for every $i \in B$. By part $(2.2)$ of Theorem 2, $(\gamma(p), \pi(p)) \in D^{*}$ and $\left(\gamma\left(p^{\prime}\right), \pi\left(p^{\prime}\right)\right) \in D^{*}$. Assume $j \in G^{>}$and let $k \in S$ be such that $j \in G_{k}^{>}$. Then, there exist $A \in F^{*}$ and $i \in B$ such that $A_{i j k}>0$. Thus, and since $(p, A)$ and $\left(p^{\prime}, A\right)$ are competitive equilibria of $M, \sum_{i^{\prime}} A_{i^{\prime} j k} \in S_{j k}\left(p_{j}\right)$ and $\sum_{i^{\prime}} A_{i^{\prime} j k} \in S_{j k}\left(p_{j}^{\prime}\right)$ imply that

$$
p_{j} \geq r_{j k} \quad \text { and } \quad p_{j}^{\prime} \geq r_{j k}
$$

By condition (CS.1) of the Complementary Slackness Theorem ,

$$
\gamma_{i}(p)+\pi_{j k}(p)-\tau_{i j k}=0
$$

and

$$
\gamma_{i}\left(p^{\prime}\right)+\pi_{j k}\left(p^{\prime}\right)-\tau_{i j k}=0
$$

Thus,

$$
\gamma_{i}(p)+\pi_{j k}(p)=\gamma_{i}\left(p^{\prime}\right)+\pi_{j k}\left(p^{\prime}\right)
$$

Since $\gamma_{i}(p) \geq \gamma_{i}\left(p^{\prime}\right)$ for every $i, \pi_{j k}\left(p^{\prime}\right) \geq \pi_{j k}(p)$ holds. By definition of $\pi_{j k}\left(p^{\prime}\right)$ and $\pi_{j k}(p)$, and since (34) holds, $\pi_{j k}\left(p^{\prime}\right)=p_{j}^{\prime}-r_{j k} \geq p_{j}-r_{j k}=\pi_{j k}(p)$. Thus, $p_{j}^{\prime} \geq p_{j}$.

$\Longleftarrow$ Assume $p_{j}^{\prime} \geq p_{j}$ for every $j \in G^{>}$. Hence, for every $i \in B$ and every $j \in G^{>}$,

$$
v_{i j}-p_{j} \geq v_{i j}-p_{j}^{\prime}
$$

Fix $i \in B$ and assume $\nabla_{i}^{>}\left(p^{\prime}\right) \neq \emptyset$. Then, there exists $j^{\prime} \in G^{>}$such that $v_{i j^{\prime}}-p_{j^{\prime}}^{\prime}>0$. By (37), $v_{i j^{\prime}}-p_{j^{\prime}}>0$, which implies that $\nabla_{i}^{>}(p) \neq \emptyset$. Hence, if $\nabla_{i}^{>}\left(p^{\prime}\right) \neq \emptyset$ there exists $j^{\prime} \in G^{>}$ such that

$$
\gamma_{i}\left(p^{\prime}\right)=v_{i j^{\prime}}-p_{j^{\prime}}^{\prime} \leq v_{i j^{\prime}}-p_{j^{\prime}}=\gamma_{i}(p)
$$

Thus, by (13), $u_{i}(p) \geq u_{i}\left(p^{\prime}\right)$. Assume now that $\nabla_{i}^{>}\left(p^{\prime}\right)=\emptyset$. Then, since by definition $0 \leq \gamma_{i}(p), \gamma_{i}\left(p^{\prime}\right)=0 \leq \gamma_{i}(p)$. Hence, by $(13), u_{i}(p) \geq u_{i}\left(p^{\prime}\right)$. Thus, for every $i \in B$, $u_{i}(p) \geq u_{i}\left(p^{\prime}\right)$. 


\section{Appendix 2: The (PLP) Has an Integer Solution}

The feasibility restrictions of the (PLP)

$$
\begin{aligned}
\sum_{j k} A_{i j k} & \leq d_{i} \quad \text { for all } i \in B \\
\sum_{i} A_{i j k} \leq q_{j k} & \text { for all }(j, k) \in G \times S
\end{aligned}
$$

can be written as $X \cdot A \leq b$. For instance, consider the case $|B|=|G|=|S|=2$. Then, (38) can be written as

$$
\left[\begin{array}{llllllll}
1 & 1 & 1 & 1 & 0 & 0 & 0 & 0 \\
0 & 0 & 0 & 0 & 1 & 1 & 1 & 1 \\
1 & 0 & 0 & 0 & 1 & 0 & 0 & 0 \\
0 & 1 & 0 & 0 & 0 & 1 & 0 & 0 \\
0 & 0 & 1 & 0 & 0 & 0 & 1 & 0 \\
0 & 0 & 0 & 1 & 0 & 0 & 0 & 1
\end{array}\right] \cdot\left[\begin{array}{c}
A_{111} \\
A_{112} \\
A_{121} \\
A_{122} \\
A_{211} \\
A_{212} \\
A_{221} \\
A_{222}
\end{array}\right] \leq\left[\begin{array}{c}
d_{1} \\
d_{2} \\
q_{11} \\
q_{12} \\
q_{21} \\
q_{22}
\end{array}\right] .
$$

Definition The matrix $X$ is totally unimodular if the determinants of all its square submatrices are equal to either $-1,0$, or 1 .

Theorem (Schrijver, 1996) If $X$ is totally unimodular then, for every integer vector $b$, the vertices of the polyhedral (the intersections of a finite number of half-spaces)

$$
\{A: X \cdot A \leq b\}
$$

have all integer coordinates.

Theorem (Schrijver, 1996) A matrix $X=\left(x_{i j}\right)_{i=1, \ldots, n}^{j=1, \ldots, m}$ is totally unimodular if:

(1) $x_{i j} \in\{-1,0,1\}$ for all $1 \leq i \leq n$ and all $1 \leq j \leq m$.

(2) Each column has at most two nonzero coefficients; namely, $\sum_{i=1}^{n}\left|x_{i j}\right| \leq 2$.

(3) Let $R=\{1, \ldots, n\}$ be the set of raws; then, there exists a partition $\left\{R_{1}, R_{2}\right\}$ of $R$ such that, for each column $j \in\{1, \ldots, m\}$ that has two non-zero coefficients,

$$
\sum_{i \in R_{1}} x_{i j}-\sum_{i \in R_{2}} x_{i j}=0 .
$$

It is easy to check that the matrix $X$ of any market satisfies conditions (1), (2), and (3) in the above theorem. Hence, $X$ is totally unimodular and thus, the (PLP) has at 
least a solution with all integer components. That $X$ satisfies (1) and (2) is immediate. To illustrate (3), consider again the case $|B|=|G|=|S|=2$. Then, $R=\{1,2,3,4,5,6\}$ and $R_{1}=\{1,2\}$ and $R_{2}=\{3,4,5,6\}$; that is,

$$
X=\left[\begin{array}{llllllll}
1 & 1 & 1 & 1 & 0 & 0 & 0 & 0 \\
0 & 0 & 0 & 0 & 1 & 1 & 1 & 1 \\
\hline \hline 1 & 0 & 0 & 0 & 1 & 0 & 0 & 0 \\
0 & 1 & 0 & 0 & 0 & 1 & 0 & 0 \\
0 & 0 & 1 & 0 & 0 & 0 & 1 & 0 \\
0 & 0 & 0 & 1 & 0 & 0 & 0 & 1
\end{array}\right]
$$




\section{Appendix 3: Lattices}

Let $X$ be a non-empty set. A partial order $\succeq$ on $X$ is a reflexive, transitive, and antisymmetric binary relation on $X$; that is, for all $x, y, z \in X, x \succeq x,[x \succeq y \succeq z] \Longrightarrow[x \succeq z]$, and $[x \succeq y$ and $y \succeq x] \Longrightarrow[x=y]$. Given a partial order $\succeq$ on $X$ and a subset $Y \subseteq X$, define the set of upper bounds of $Y$ as $u b_{\succeq} Y=\{x \in X \mid x \succeq y$ for all $y \in Y\}$ and the least upper bound of $Y$ as $l u b_{\succeq} Y=\bar{y}$, where $\bar{y} \in u b_{\succeq} Y$ and, for all $y \in u b_{\succeq} Y, y \succeq \bar{y}$. Similarly, define the set of lower bounds of $Y$ as $l b_{\succeq} Y=\{x \in X \mid y \succeq x$ for all $y \in Y\}$ and the largest lower bound of $Y$ as $l l b_{\succeq} Y=\underline{y}$ where $\underline{y} \in l b_{\succeq} Y$ and, for all $y \in l b_{\succeq} Y, \underline{y} \succeq y$. Given a partial order $\succeq$ on $X$, define the binary operations $\vee$ and $\wedge$ on $X$ as follows: for $x, y \in X, x \vee y=l u b_{\succeq}\{x, y\}$ and $x \wedge y=l l b_{\succeq}\{x, y\}$. Observe that, in general, $l u b_{\succeq}\{x, y\}$ and $l l b_{\succeq}\{x, y\}$ may not exist; however, by the antisymmetry of $\succeq$, if they exist, they are unique.

Definition The four-tuple $(X, \succeq, \vee, \wedge)$ is a lattice if, for all $x, y \in X, l u b_{\succeq}\{x, y\}$ and $l l b_{\succeq}\{x, y\}$ exist. A set $X$ has a lattice structure if $(X, \succeq, \vee, \wedge)$ is a lattice for some $\succeq, \vee$, and $\wedge$.

A lattice $(X, \succeq, \vee, \wedge)$ is complete if for any subset $Y \subseteq X, l u b_{\succeq} Y \in X$ and $l l b_{\succeq} Y \in X$.

Observe that since the binary operations $\vee$ and $\wedge$ follow from the partial order $\succeq$ there is some redundancy in the notation $(X, \succeq, \vee, \wedge)$ of a lattice. However, it is useful (and common) to refer simultaneously to the partial order and to the two binary operations because there is an (equivalent) algebraic approach where, instead of starting from the partial order $\succeq$, one can start from two binary operations on $X$ as follows. A join $\vee$ and a meet $\wedge$ on $X$ are two idempotent, commutative, associative, and absorbing binary operations on $X$; that is, for all $x, y, z \in X, x \vee x=x$ and $x \wedge x=x, x \vee y=y \vee x$ and $x \wedge y=y \wedge x, x \vee(y \vee z)=(x \vee y) \vee z$ and $x \wedge(y \wedge z)=(x \wedge y) \wedge z$, and $x=(x \vee y) \wedge x$ and $x=(x \wedge y) \vee x$. Given a join $\vee$ and a meet $\wedge$ on $X$, define the partial orders $\succeq^{\vee}$ and $\succeq^{\wedge}$ on $X$ as follows: for all $x, y \in X$,

$$
x \succeq^{\vee} y \text { if and only if } x=x \vee y
$$

and

$$
x \succeq^{\wedge} y \text { if and only if } y=y \wedge x .
$$

Indeed, both approaches are equivalent in the sense that the partial orders $\succeq^{\vee}$ and $\succeq^{\wedge}$ obtained from $\vee$ and $\wedge$ are the same and coincide with $\succeq$ (i.e., the partial order from which 
$\vee$ and $\wedge$ are defined)..$^{12}$

We use this second approach to show in Theorem 3 that the set $P^{* *}$ has a lattice structure with the following natural join and meet. Let $p, p^{\prime} \in P^{* *}$. Define $p \vee p^{\prime} \equiv \check{p}=\left(\check{p}_{j}\right)_{j \in G}$ and $p \wedge p^{\prime} \equiv \hat{p}=\left(\hat{p}_{j}\right)_{j \in G}$ as follows. For each $j \in G$,

$$
\check{p}_{j}=\max \left\{p_{j}, p_{j}^{\prime}\right\}
$$

and

$$
\hat{p}_{j}=\min \left\{p_{j}, p_{j}^{\prime}\right\}
$$

\footnotetext{
${ }^{12}$ See Grätzer (2003).
} 